\title{
ON THE DECOMPOSITION NUMBERS OF THE FINITE GENERAL LINEAR GROUPS ${ }^{1}$
}

BY

\author{
RICHARD DIPPER
}

\begin{abstract}
Let $G=\mathrm{GL}_{n}(q), q$ a prime power, and let $r$ be an odd prime not dividing $q$. Let $s$ be a semisimple element of $G$ of order prime to $r$ and assume that $r$ divides $q^{\operatorname{deg}(\Lambda)}-1$ for all elementary divisors $\Lambda$ of $s$. Relating representations of certain Hecke algebras over symmetric groups with those of $G$, we derive a full classification of all modular irreducible modules in the $r$-block $B_{s}$ of $G$ with semisimple part $s$. The decomposition matrix $D$ of $B_{s}$ may be partly described in terms of the decomposition matrices of the symmetric groups corresponding to the Hecke algebras above. Moreover $D$ is lower unitriangular. This applies in particular to all $r$-blocks of $G$ if $r$ divides $q-1$. Thus, in this case, the $r$-decomposition matrix of $G$ is lower unitriangular.
\end{abstract}

Introduction. The modular representation theory of finite groups of Lie type defined over a field of characteristic $p$ is naturally divided into the cases of equal characteristic $r=p$ and unequal characteristic $r \neq p$. This paper begins the study of decomposition numbers in the unequal characteristic case of the finite general linear groups when $r>2$.

Let $G$ be the full linear group of degree $n$ over $\mathrm{GF}(q)$ and $2 \neq r$ be a prime not dividing $q$. Let $s \in G$ be semisimple and consider the geometric conjugacy class $(s)^{G}$ of irreducible characters corresponding to the $G$-conjugacy class of $s$. Using basic properties of the Deligne-Lusztig operators introduced in [7] we will determine a parabolic subgroup $P=P_{s}$ of $G$ and a cuspidal irreducible character of $\chi$ of $P$ such that $(s)^{G}$ is just the set of constituents of $\chi^{G}$, the induced character. Let $\{\bar{R}, R, K\}$ be an $r$-modular system for $G$ and $M$ a $K P$-module affording $\chi$. Then $\operatorname{End}_{K G}\left(M^{G}\right)$ is called the Hecke algebra of $M$ (compare [5]) and has been calculated in a more general context by R. B. Howlett and G. I. Lehrer in [12]. It turns out that End $_{K G}\left(M^{G}\right) \cong K[W]$, the Hecke algebra of $W$, where $W=W_{s}$ denotes the Weyl group of $C_{G}(s)$ (the centralizer of $s$ in $G$ ) (compare $\left.[1,2,4,13]\right)$. In particular, $K[W]$ has a basis $\left\{T_{w} \mid w \in W\right\}$ such that the structure constants of the multiplication are all contained in $R \subseteq K$. So let $R[W]$ be the $R$-order in $K[W]$ generated by $\left\{T_{w} \mid w \in W\right\}$ and $\bar{R}[W]=\bar{R} \otimes_{R} R[W]$. Choosing a special $R P$-lattice $S$ in $M$, and using the classification of the $r$-blocks of $R G$ given by P. Fong and B. Srinivasan in

Received by the editors July 25, 1983 and, in revised form, October 18, 1983, May 23, 1984 and September 1, 1984.

1980 Mathematics Subject Classification. Primary 20C20; Secondary 20 G40.

${ }^{1}$ This work was supported by the Deutsche Forschungsgemeinschaft. 
their fundamental paper [9], we will show that $\operatorname{End}_{\tilde{R} G}\left(\tilde{R} S^{G}\right) \cong \tilde{R}[W]$ for every choice of $\tilde{R} \in\{\bar{R}, R, K\}$ if $s \in G$ satisfies certain conditions (Hypothesis 2.4), which are fulfilled, in particular, if the order of $s$ is prime to $r$. This generalizes parts of [12]. We define a certain subalgebra $A$ of $R G$, which does not arise from a subgroup of $G$, and which will play the role of an inertia algebra of $S$ in $G$. Using this algebra we will prove a homological lemma of Clifford type (Lemma 3.7), which enables us to connect the submodule structure of $S^{G}$ and $R[W]$. It is a well-known theorem that $K[W] \cong K W$, the usual group algebra of $W$ over $K$. This does not remain true, in general, if we replace $K$ by $R$ or $\bar{R}$. But there is an important special case where $\bar{R}[W] \cong \bar{R} W$ canonically, namely if $r$ divides $q^{\operatorname{deg} \Lambda_{-1}}$ for all elementary divisors of $s$, and we may apply then the known facts of the representation theory of symmetric groups.

Thus let $s$ be a semisimple $r^{\prime}$-element of $G$ and assume that $r$ divides $q^{\operatorname{deg}} \Lambda_{-}-1$ for all elementary divisors $\Lambda$ of $s$. By [9] there is just one $r$-block $B$ of $R G$ with semisimple part $s$ and the union of the geometric conjugacy classes $(s y)^{G}$, where $y$ runs through a Sylow $r$-subgroup $D$ of $C_{G}(s)$, is just the set of irreducible characters in $B$. Note that $r$ divides $q^{\operatorname{deg} \Gamma}-1$ for all elementary divisors $\Gamma$ of $s y$, too. We define a sequence $1=y_{1}, \ldots, y_{k}$ in $D$ such that $t_{i}=s y_{i}$ satisfies Hypothesis 2.4 , so we may apply the results of $\S \S 3$ and 4 to the geometric classes $\left(t_{i}\right)^{G}$. This leads to a full classification of the irreducible $\bar{R} G$-modules contained in $B$. We obtain parts of the decomposition matrix of $B$ in terms of the decomposition matrices of $W_{t_{i}}(1 \leq i \leq k)$, the Weyl group of $C_{G}\left(t_{i}\right)$. In particular, we show that the irreducible characters and irreducible Brauer characters in $B$ may be ordered such that the decomposition matrix of $B$ has generalized lower triangular form with one's in the main diagonal, a fact known for the decomposition matrices of symmetric groups. As a corollary we get that a cuspidal irreducible character is an irreducible Brauer character if it is restricted to the set of $r^{\prime}$-elements of $G$ for all primes $r$ which divide $q-1$. In fact, it seems to be likely that this holds in general.

Throughout this paper we will use the standard notation $\mathbf{Z}, \mathbf{R}$, etc. for the integers, real numbers respectively. If not stated otherwise, all occurring modules are finitely generated right modules. All rings are $R$-free, finite dimensional $R$-algebras for some domain $R$. For an $R$-algebra $A$ and an $A$-module $M$, we denote the Jacobson radical of $M$ by $J(M)$. The factor module $M / J(M)$ is called the head of $M$.

The author is very indebted to P. Fong and B. Srinivasan for many helpful suggestions and discussions.

1. First let us fix some notation. Throughout this paper let $q$ be a power of some fixed prime $p$. Let $F=\mathrm{GF}(q)$ be the finite field with $q$ elements and $G=\operatorname{GL}_{n}(q)$ the full linear group of degree $n$ over $F$ for a fixed natural number $n$. Let $R$ be a discrete rank one valuation ring with maximal ideal $J(R)$ (the Jacobson radical of $R$ ), and assume that the quotient field $K$ of $R$ has characteristic zero, and $\bar{R}=$ $R / J(R)$ is a field of characteristic $r>0$ for some odd prime $r \neq p$. For an $R$-module $V$ we will write $\bar{R} V$ or $\bar{V}$ for $\bar{R} \otimes_{R} V \cong V / J(R) V$, and $K V$ for $K \otimes_{R} V$. Furthermore, we will choose $R$ such that $K$ and $\bar{R}$ are both splitting fields for all subgroups of $G$. 
Next we will describe briefly the semisimple conjugacy classes and the irreducible characters of $G$, where we mostly adopt the notation of [9].

So let $X$ be an indeterminant and let $\mathscr{F}$ be the set of monic irreducible polynomials in $X$ over $F$, different from the polynomial $p(X)=X$. For $\Lambda \in \mathscr{F}, s \in G$ and $k \in \mathrm{N}$ let $\operatorname{deg} \Lambda$ be the degree of $\Lambda, k(\Lambda) \in \mathrm{Gl}_{k \cdot \operatorname{deg} \Lambda}(q)$ the matrix direct sum of $k$ copies of the companion matrix of $\Lambda$ and $m_{\Lambda}(s)$ the multiplicity of $\Lambda$ as elementary divisor of $s$. If $s \in G$ is semisimple, all elementary divisors of $s$ are contained in $\mathscr{F}$, hence $s$ is conjugate in $G$ to $\prod_{\Lambda \in \mathscr{F}} m_{\Lambda}(s)(\Lambda)$, where $\Pi$ stands for matrix direct sum, and factors of multiplicity zero have to be dropped. This is just the rational canonical form of $s$ and is uniquely determined up to the order of the factors. So let us make the following convention: First we order $\mathscr{F}$ totally such that $\operatorname{deg} \Lambda<\operatorname{deg} \Gamma$ $(\Lambda, \Gamma \in \mathscr{F})$ implies $\Lambda<\Gamma$. All occurring products will be taken in this fixed order, and, in the following, $\Pi, \Sigma, \cup$, etc. always mean $\Pi_{\Lambda \in \mathscr{F}}, \Sigma_{\Lambda \in \mathscr{F}}, \cup_{\Lambda \in \mathscr{F}}$, etc. With this convention

$$
\left\{\Pi k_{\Lambda}(\Lambda) \mid 0 \leq k_{\Lambda} \in \mathbf{N}, \Sigma k_{\Lambda} \cdot \operatorname{deg} \Lambda=n\right\}
$$

is a full set of representatives of semisimple conjugacy classes of $G$. For semisimple $s=\prod m_{\Lambda}(s)(\Lambda)$ in $G$, the centralizer $C_{G}(s)$ of $s$ in $G$ is given by

$$
C_{G}(s)=\prod \mathrm{GL}_{m_{\Lambda}(s)}\left(q^{\operatorname{deg} \Lambda}\right),
$$

where we identify $\operatorname{GF}\left(q^{\operatorname{deg} \Lambda}\right)$ with the subring of the (deg $\left.\Lambda \times \operatorname{deg} \Lambda\right)$-matrix ring over $F$ generated by $F$ and $(\Lambda)$.

For the notation of maximal tori, regular, parabolic and Levi subgroups we refer to [15]. In particular, recall that the set of all diagonal matrices in $G$ is a maximal torus of $G$, the standard split torus $T_{0}$. The Weyl group $W_{G}=W_{0}$ of $G$ is defiped to be $N_{G}\left(T_{0}\right) / T_{0}$, where $N_{G}\left(T_{0}\right)$ denotes the normalizer of $T_{0}$ in $G$. In the following, we identify always $W_{0}$ with the subgroup of all permutation matrices in $G$, which is canonically isomorphic to $S_{n}$, the symmetric group on $n$ letters (permuting the natural basis of the natural module for $G$ ). The $G$-conjugacy classes of maximal tori are in bijection with the conjugacy classes of $W_{0}$, hence with partitions of $n$. More precisely, to the partition $\lambda=\left(1^{r_{1}}, \ldots, n^{r_{n}}\right)$ of $n$ we may construct a representative $T_{\lambda}$ of the corresponding $G$-conjugacy class of maximal tori in the following way: First, for $1 \leq i \leq n$ choose an irreducible polynomial $\Lambda \in \mathscr{F}$ whose roots generate $\operatorname{GF}\left(q^{i}\right)$ over $F=\mathrm{GF}(q)$. Then, as above, $F$ and $(\Lambda)$ generate a subalgebra $F_{i}$ of the $i \times i$-matrix over $\mathscr{F}$ isomorphic to $\operatorname{GF}\left(q^{i}\right)$. Now define $T_{\lambda}=\left(F_{n}{ }^{*}\right)^{r_{n}} \times \cdots \times\left(F_{1}{ }^{*}\right)^{r_{1}}$ $\leq G$ (matrix direct sum), where $F_{i}{ }^{*}$ denotes the multiplicative group of $F_{i}(1 \leq i \leq n)$. In particular $T_{0}=T_{\lambda}$ for $\lambda=\left(1^{n}\right)$. A maximal torus corresponding to $\lambda=(n)$ is called a Coxeter torus. If $T$ is a maximal torus of $G$, the Weyl group $W(T)=W_{G}(T)$ of $T$ (in $G$ ) is defined to be $N_{G}(T) / T$. If $T$ corresponds to $\lambda=\left(\lambda_{1} \geq \lambda_{2} \geq \cdots \geq\right.$ $\left.\lambda_{k}\right)$, then $W(T)$ is isomophic to $C_{W_{0}}\left(w_{\lambda}\right)$, the centralizer of $w_{\lambda} \in W_{0}=W_{G}\left(T_{0}\right)$ in $W_{0}, w_{\lambda}=w_{1} \cdots w_{k}$, where $w_{i} \in W_{0}$ is defined to be the cycle $\left(j_{i}+1, \cdots, j_{i+1}\right)$ in $W$ with $j_{i}=\sum_{m=1}^{i-1} \lambda_{m}$. Of course $C_{W_{0}}\left(w_{\lambda}\right)$ is the semidirect product of $\prod_{j=1}^{k}\left\langle w_{i}\right\rangle$ and $W_{\lambda} \cong S_{r_{i}} \times \cdots \times S_{r_{n}}\left(\lambda=\left(\lambda_{1} \geq \cdots \geq \lambda_{k}\right)=\left(1^{r_{i}}, \ldots, n^{r_{n}}\right)\right)$, generated by the involutions 
$\left(j_{i}+1, j_{m+1}+1\right) \cdots\left(j_{i+1}, j_{m+1}\right)$, where $1 \leq i, m \leq k$ are such that $\lambda_{i}=\lambda_{m}$. If $T=T_{\lambda}$ is constructed as above, then it can easily be seen that $T$ has a complement in $N_{G}(T) \leq G$ which is the semidirect product of a subgroup of $\prod_{i=1}^{n} G_{i}(q)^{r_{i}} \leq G$ isomorphic to $\prod_{i=1}^{k}\left\langle w_{i}\right\rangle$ by $W_{j}$ (in fact conjugate to $\prod_{i=1}^{k}\left\langle w_{i}\right\rangle$ in $\operatorname{GL}_{n}\left(q^{m}\right) \geq G$ for a suitable $m \in \mathbf{N})$. Of course $N_{G}(T) / T$ is isomorphic to this complement. Abusing notation, we call this complement Weyl group of $T$, too. In addition, throughout this paper, $W_{G}(T)$ always denotes the complement of $T$ in $N_{G}(T)$, whereas we shall denote the Weyl group of $T$ in $G$ in the original sense by $N_{G}(T) / T$. So $W_{G}(T) \leq G$. Note in particular that $W_{0}=W_{G}\left(T_{0}\right)$ is the set of permutation matrices in $G$.

Now let $L$ be a regular subgroup of $G$. Let $R_{L}^{G}$ be the additive operator from $X(L)$ to $X(G)$ defined in the Deligne-Lusztig theory, where $X(L)$ and $X(G)$ are the character rings of $L$ and $G$ respectively over $K$, following the notation used in [9] (there is no harm in replacing the algebraically closed field in [9] by $K$ ). For the definition and basic properties of these operators we refer to [15].

In [10] J. A. Green has classified the irreducible characters of $G$. In the DeligneLusztig theory these are parametrized by $G$-conjugacy classes of pairs $(s, \mu)$ where $s \in G$ is semisimple and $\mu=\prod \mu_{\Lambda}$ (cartesian product), with $\mu_{\Lambda}$ a partition of $m_{\Lambda}(s)$. So for semisimple $s$ in $G$, let $M_{s}$ be the set of all such products of partitions of $m_{\Lambda}(s)$. The corresponding character of $G$ will be denoted by $\chi_{s, \mu}$.

In [9] P. Fong and B. Srinivasan have classified the blocks of $R G$ with their defect groups. Furthermore, they determined for each $r$-block the characters contained in it. Following the notation introduced in [9] we have a bijecton $B \rightarrow B_{s, \lambda}$ between the blocks $B$ of $R G$ and $G$-conjugacy classes $\left(s^{G}, \lambda\right)$ of pairs $(s, \lambda)$, where $s$ is a semisimple $r^{\prime}$-element of $G$ and $\lambda$ the unipotent factor of $B$ as defined in [9]. $\lambda$ can be derived from elements of $M_{s}$ in terms of hooks and cores. We will need the following special case. Let $\Lambda \in \mathscr{F}$ be such that $\operatorname{deg} \Lambda=n$ and all roots of $\Lambda$ have order prime to $r$. Then $s=(\Lambda) \in G$ is a semisimple $r^{\prime}$-element. It can easily be seen that there is just one possible unipotent factor associated with $s$, say $\lambda$, and we may write $B_{s}=B_{s, \lambda}$. Furthermore, it follows from [9] that $B_{s}$ is a block with cyclic defect group and inertial index one. So there is just one irreducible $R G$-module $M$ in $B_{s}$, and every irreducible $K G$-module in $B_{s}$, when reduced modulo $r$, is isomorphic to $M$. Note that all definitions and formulas above (and the following as well) may easily be generalized to finite direct products of finite full linear groups.

Now let $s \in G$ be semisimple, and consider $L_{0}=C_{G}(s)$. The center of $L_{0}$ is isomorphic to the multiplicative group of linear characters of $L_{0}$, and this isomorphism may be chosen such that $\widehat{s^{g}}=\hat{s} g$ for all $g \in N_{G}\left(L_{0}\right)$, where the linear character corresponding to $s$ will be denoted by $\hat{s}$.

$L_{0}$ is conjugate in $G$ to $\prod \mathrm{GL}_{m_{1}(s)}\left(q^{\operatorname{deg} \Lambda}\right)$. In particular, the Weyl group $W_{L_{0}}=$ $W_{s}=W$ of $L_{0}$ is isomorphic to $\prod S_{m_{\Lambda}(s)}$. Consequently, the $L_{0}$-conjugacy classes of maximal tori of $L_{0}$ correspond bijectively to $M_{s}$. For $\mu=\Pi \mu_{\Lambda} \in M_{s}$ define the partition $\tilde{\mu}$ of $n$ in the following way: First, for $\Lambda \in \mathscr{F}, \mu_{\lambda}=\left(1^{k_{1}}, 2^{k_{2}}, \ldots\right)$, define the partition $\operatorname{deg} \Lambda \cdot \mu_{\Lambda}$ of $\operatorname{deg} \Lambda \cdot m_{\Lambda}(s)$ setting

$$
\operatorname{deg} \Lambda \cdot \mu_{\Lambda}=\left((\operatorname{deg} \Lambda)^{k_{1}},(2 \operatorname{deg} \Lambda)^{k_{2}}, \ldots\right) .
$$


Let $r_{i, \lambda}$ be the exponent of $i$ in $\operatorname{deg} \Lambda \cdot \mu_{\Lambda}$ for $1 \leq i \leq \operatorname{deg} \Lambda \cdot m_{\Lambda}(s)$, and $r_{i, \Lambda}=0$ for $\operatorname{deg} \Lambda \cdot m_{\Lambda}(s)+1 \leq i \leq n$. Set $r_{i}=\sum r_{i, \Lambda}$, and define $\tilde{\mu}$ setting $\tilde{\mu}:=\left(1^{r_{1}}, \ldots, n^{r_{n}}\right)$. It can easily be seen that a maximal torus of $L_{0}$ corresponding to $\mu \in M_{s}$ is also a maximal torus of $G$, contained in the $G$-conjugacy class of maximal tori of $G$ corresponding to $\tilde{\mu}$. The map $\sim: \mu \rightarrow \tilde{\mu}$ is not injective in general (i.e. there are maximal tori in $L_{0}$, which are not conjugate in $L_{0}$ but in $\left.G\right)$, but if $\mu=\Pi\left(1^{m_{\Lambda}(s)}\right) \in$ $M_{s}, \nu \in M_{s}$ and $\mu \neq \nu$, then $\tilde{\mu} \neq \tilde{\nu}$. In other words, the maximal split torus $T=T_{s}$ ( $=T_{\mu}$ ) of $L_{0}$ is not conjugate in $G$ to $T_{\tilde{\nu}}$.

It is well known (see e.g. [15]) that there is a bijection between the set of all irreducible characters of $W=W_{s}$ and $M_{s}$, and between the set of conjugacy classes of $W$ and $M_{s}$ as well. So, for $\mu, \lambda \in M_{s}$, let $\phi^{\mu}(\lambda)$ be the value of the irreducible character of $W$ corresponding to $\mu$ on the conjugacy class of $W$ corresponding to $\lambda$. Furthermore, let $\hat{\lambda}$ be the number of elements in this class. Finally denote the restriction of $\hat{s}$ to $T_{\mu}, \mu \in M_{s}$, again by $\hat{s}$. Now define the generalized character $\chi^{s, \mu}$ of $G$ by

$$
\chi^{s, \mu}=\frac{1}{|W|} \sum_{\lambda \in M_{s}} \hat{\lambda} \phi^{\mu}(\lambda) R_{T_{\lambda}}^{G}(\hat{s}) .
$$

Then $\chi^{s, \mu}$ is up to sign the irreducible character $\chi_{s, \mu}$ of $G$ (see e.g. $[9,1.18]$ ).

Note that we may replace $s$ by $s^{g}=g^{-1} s g$ for $g \in G$. So we may assume in the following that $s$ is given in the rational canonical form. In particular, $s \in T$, $L_{0}=C_{G}(s)=\prod \mathrm{GL}_{m_{\Lambda}(s)}\left(q^{\operatorname{deg} \Lambda}\right) \leq G$ canonically, and $W=W_{s} \leq W_{0}$.

\subsection{LEMMA. $R_{T}^{G}(\hat{s})=\sum_{\mu \in M_{s}} \phi^{\mu}(1) \chi^{s, \mu}$.}

Proof. Let $(\cdot, \cdot)=(\cdot, \cdot)_{G}$ be the usual scalar product in $X(G)$. By the orthogonality relations for the Deligne-Lusztig operators (see e.g. $[9,1.6]$ ) the following holds:

$$
\left(R_{T_{\hat{\lambda}}}^{G}(\hat{s}), R_{T}^{G}(\hat{s})\right)=0 \quad \text { for } \Pi\left(1^{m_{\Lambda}(s)}\right) \neq \lambda \in M_{s},
$$

and

$$
\left(R_{T}^{G}(\hat{s}), R_{T}^{G}(\hat{s})\right)=\left|C_{W_{G}(T)}(\hat{s})\right|=\left|C_{W_{G}(T)}(s)\right|=|W| ;
$$

for, since $T$ is abelian and $s \in T$,

$$
C_{N_{G}(T) / T}(s) \cong C_{N_{G}(T)}(s) / T=\left(N_{G}(T) \cap C_{G}(s)\right) / T=N_{L_{0}}(T) / T \cong W .
$$

Consequently

$$
\begin{aligned}
\left(\chi^{s, \mu}, R_{T}^{G}(\hat{s})\right) & =|W|^{-1} \sum_{\lambda \in M_{s}} \hat{\lambda}^{\mu}(\lambda)\left(R_{T_{\lambda}}^{G}(\hat{s}), R_{T}^{G}(\hat{s})\right) \\
& =|W|^{-1} \phi^{\mu}(1)\left(R_{T}^{G}(\hat{s}), R_{T}^{G}(\hat{s})\right)=\phi^{\mu}(1) .
\end{aligned}
$$

Now $\sum_{\mu \in M_{s}}\left(\phi^{\mu}(1)\right)^{2}=|W|$. This proves the lemma.

Now let $L=L_{s}=\Pi\left(\mathrm{GL}_{\operatorname{deg} \Lambda}(q)\right)^{m_{\Lambda}(s)}$, embedded in $G$ in the obvious way. Then $s \in L$, in fact the standard split torus $T=T_{s}$ of $L_{0}$ (which contains $s$ ) is a Coxeter torus of $L$ and $C_{L}(s)=L \cap L_{0}=T$. Of course, $L$ is a Levi complement of some parabolic subgroup $P=L U$ with Levi kernel $U=U_{s}$, and we can choose $P=L U_{0}$, 
where $U_{0}$ denotes the subgroup of all unipotent upper triangular matrices in $G$. Every $L$-module $M$ (over $R, \bar{R}$ or $K$ ) may be considered as $P$-module via the epimorphism $P \rightarrow L$. The corresponding $P$-module will be denoted by $\tilde{M}$. Similarly, for a character $\chi$ of $L$ let $\tilde{\chi}$ be the pull back of $\chi$ to $P$. Furthermore, if $H \leq G$ and if $M$ is an $H$-module, $N$ a $G$-module, let $M^{G}$ be the induced and $N_{H}$ the restricted module.

1.2 LEMMA. $R_{T}^{G}(\hat{s})={\widetilde{\left(R_{T}^{L}(s)\right.}}^{G}$.

Proof. [15, 6.24].

1.3. LEMMA. $R_{T}^{L}(\hat{s})$ is up to sign an irreducible cuspidal character of $L$.

Proof. $C_{G}(s) \cap W_{L}(T) \leq L \cap L_{0}=T$, hence the centralizer of $s$ (therefore of $\hat{s}$ too) in $N_{L}(T) / T$ is trivial, i.e. $\hat{s}$ is a regular character of $T \leq L$. So $\pm R_{T}^{L}(\hat{s})$ is irreducible by $[15,6.19]$. Of course $\left(R_{T}^{L}(\hat{s}), R_{\tilde{T}}^{L}(\theta)\right)_{L}=0$ for every maximal torus $\tilde{T}$ of $L$ not conjugate to $T=T_{s}$ in $L[15,6.14]$, and for any character $\theta$ of $\tilde{T}$. So $\pm R_{T}^{L}(\hat{s})$ is cuspidal by $[15,6.25]$.

Next let $L(\Lambda)=\mathrm{GL}_{\operatorname{deg} \Lambda}(q) \quad(\Lambda \in \mathscr{F})$; thus $L=\Pi L(\Lambda)^{m_{\Lambda}(s)}$. Let $s=$ $\Pi m_{\Lambda}(s)(\Lambda), \hat{s}=\Pi \overline{(\Lambda)}^{m_{\Lambda}(s)}$ (tensor product), and $T=\Pi T(\Lambda)^{m_{\Lambda}(s)}$ be the corresponding decompositions of $s, \hat{s}$ and $T_{s}=T$ respectively. Then $T(\Lambda)$ is a Coxeter torus of $L(\Lambda)$ and $C_{L(\Lambda)}((\Lambda))=T(\Lambda)$. Applying 1.3 to $L(\Lambda)$ we conclude that $R_{T(\Lambda)}^{L(\Lambda)}(\widehat{(\Lambda)})$ is up to sign an irreducible character of $L(\Lambda)$. Let $S(\Lambda)$ be an $R L(\Lambda)$-lattice in the irreducible $K L(\Lambda)$-module affording this irreducible character, and set $S_{0}=\Pi S(\Lambda)^{m_{\Lambda}(s)}$ (tensor product).

1.4. LemMa. $K S_{0}$ is an irreducible $K L$-module. The character afforded by $K S_{0}$ is up to $\operatorname{sign} R_{T}^{L}(\hat{s})$.

Proof. Note first that by the choice of the isomorphism between the center of $L_{0}=C_{G}(s)$ and the multiplicative group of linear characters of $L_{0}$, in fact $\hat{s}=$ $\Pi\left(\overline{(\Lambda)}^{m_{\Lambda}(s)}\right.$. As an easy consequence from the Künneth formula for 1-adic cohomology (see e.g. $[15,5.9])$ we now get that

$$
R_{T}^{L}(\hat{s})=\Pi\left(R_{T(\Lambda)}^{L(\Lambda)}(\widehat{(\Lambda)})\right)^{m_{\Lambda}(s)}
$$

Now the lemma follows easily from 1.3.

Let $S=\tilde{S}_{0}$, i.e. the pull back of $S_{0}$ to $P$. Then $1.1,1.2$ and 1.4 imply

1.5. Lemma. $K S^{G}=\sum_{\mu \in M_{s}} \phi^{\mu}(1) N_{\mu}$, where $N_{\mu}, \mu \in M_{s}$, is an irreducible $K G$-module affording $\chi_{s, \mu}$.

In particular, counting multiplicities, this implies the following well-known corollary.

1.6. CoROllaRY. The endomorphism ring $\operatorname{End}_{K G}\left(K S^{G}\right)$ of $K S^{G}$ is isomorphic to the group algebra $K W_{s}$.

Throughout this paper we will keep the notation introduced above. In particular, $s \in G$ will always denote a semisimple element of $G$, given in the rational canonical 
form, and $M_{s}, W=W_{s}, T=T_{s}, L=L_{s}, P=P_{s}=L U, U=U_{s}$ and $S$ will be defined as above. If necessary, we will use the index $s$. Furthermore let $b=b_{s}$ be the block of $R P$ containing $S$ and $e=e_{s}$ the block idempotent of $b$. By general arguments (see e.g. [8, 4.3]), the blocks of $R L$ are just those blocks of $R P$ which contain $U$ in their kernel, because $U$ is a normal $r^{\prime}$-subgroup of $P$. In particular, $U$ is in the kernel of $b$ and all irreducible $K P$-modules in $b$ contain $U$ in their kernel. So we may consider $b$ as the block of $R L$ and decompose it in $b=\Pi b_{\Lambda}^{m_{\Lambda}(s)}$ corresponding to the decomposition of $L$. If $e_{0}$ is the block idempotent of $b$ considered as the block of $R L$, then $e=e_{0} f$ with $f=f_{s}=|U|^{-1} \sum_{u \in U} u$. Note that $f \in \tilde{R} G$ for every choice of $\tilde{R} \in\{\bar{R}, R, K\}$.

2. For the moment we will assume in addition that the order of $s \in G$ is prime to $r$. For $\Lambda \in \mathscr{F}$ consider the block $b_{\Lambda}$ of $L(\Lambda)$. As remarked in the first section there is just one block, namely $b_{\Lambda}$, of $R L(\Lambda)$ with semisimple part $(\Lambda) \in L(\Lambda)$, because there is only one possible unipotent factor associated with $(\Lambda)$. Furthermore, $b_{\Lambda}$ has cyclic defect group and inertial index 1 , so $\overline{S(\Lambda)}=\bar{R} S(\Lambda)$ is the unique irreducible $\bar{R} L(\Lambda)$-module in $b_{\Lambda}$. This implies immediately

2.1. Lemma. Let $N$ be an irreducible KP-module in $b$, and $S_{1}$ and $R P$-lattice in $N$. Then $\bar{S}_{1}=\bar{R} S_{1} \cong \bar{R} S$, and $\bar{R} S$ is the unique irreducible $\bar{R} P$-module in $b$.

Recall that the set of characters $\chi_{t, \mu}$, where $t$ is a fixed semisimple element of $G$ and $\mu$ runs through $M_{t}$, is called the geometric conjugacy class corresponding to $t$ and is denoted by $(t)^{G}$. Assume that $\chi \in(t)^{L}$ and $\chi \in b$. Then [9, 7A] implies in particular that $t=s y$, for some $r$-element $y \in T_{s}$. So we conclude

2.2. Lemma. Let $\psi$ be an irreducible character of $P$ contained in $b$. Then $\psi=\chi$ for some $\chi \in(s y)^{L}$ and some r-element $y \in T$.

2.3. LemMA. Let $V$ be an irreducible KP-module contained in $b$. Assume that $V$ occurs as the constituent of $K S_{P}^{G}$. Then $V \cong K S$.

Proof. Let $\psi$ be the character afforded by $V$. Then, by $2.2, \psi=\chi$ and $\chi \in(s y)^{L}$ for some $y \in T$. Let $t=s y$. Then $C_{L}(t)=C_{L}(s)=T$. In particular, $C_{L}(t)$ is a direct product of multiplicative groups of fields, so, considered as a product of linear groups, its Weyl group is trivial, and $(t)^{L}$ contains only one element, namely $\chi$. Therefore $\chi= \pm R_{T}^{L}(\hat{t})$, and by $[15,6.24], \psi^{G}= \pm R_{T}^{G}(\hat{t})$. Obviously the multiplicity of $V$ in $K S_{P}^{G}$ is just $\left|\left(\psi, R_{T}^{G}(\hat{s})\right)_{P}\right|$. By Frobenius reciprocity and [15, 6.24], $\left(\psi, R_{T}^{G}(\hat{s})\right)_{P}=\left(\tilde{\chi}^{G}, R_{T}^{G}(\hat{s})\right)_{G}= \pm\left(R_{T}^{G}(\hat{t}), R_{T}^{G}(\hat{s})\right)=\left|\left\{g \in N_{G}(T) / T \mid t^{g}=s\right\}\right|$. Now, if $y \neq 1$, the orders of $s$ and $t$ are different and they cannot be conjugate by an element of $N_{G}(T) / T$. Thus $y=1$, that is $t=s$ and $\chi \in(s)^{L}$. Because $(s)^{L}$ contains exactly one element, namely the character afforded by $K S$, we conclude $V \cong K S$ as desired.

Now let $s \in G$ be again an arbitrary semisimple element.

2.4. Hypothesis. Assume that $s$ satisfies the following conditions:

(i) $\bar{R} S$ is an irreducible $\bar{R} P$-module.

(ii) Let $V \in b$ be an irreducible constituent of $K S_{P}^{G}$. Then $V \cong K S$. 
We have seen in 2.1 and 2.3 that $s \in G$ satisfies 2.4 as long as its order is prime to $r$. So the results of the following sections are always true in this case, but we will need it also for some special elements of the form $s y$, where $s$ is a semisimple $r^{\prime}$-element of $G$ and $y$ is an $r$-element of $C_{G}(s)$. We have to verify 2.4 in these cases. We will prove later that (i) is always true if $r$ divides $q-1$; in fact, it seems to be true in general, whereas (ii) is not satisfied by all semisimple elements of $G$.

3. Let $s \in G$ be semisimple, given in the rational canonical form, and assume 2.4. Now $W$, permuting the equal factors of $s=\prod m_{\Lambda}(s)(\Lambda)$, normalizes $L$. On the other hand $L \cap W \leq L \cap C_{G}(s)=T, W \cap T=(1)$ implies that $H=L W \leq G$ is a semidirect product.

Let $\tilde{R} \in\{\bar{R}, R, K\}$. Recall that $\tilde{R} S_{0}=\Pi \tilde{R} S(\Lambda)^{m_{\cdot}(s)}$. Now $w \in W$ acts as permutation on the factors of $\tilde{R} S(\Lambda)^{m_{\lambda}(s)}$ for each $\Lambda \in \mathscr{F}$. So $\tilde{R} S_{0}{ }^{w} \cong \tilde{R} S_{0}$ for all $w \in W$, and obviously the $\tilde{R} L$-isomorphisms $t_{w}: \tilde{R} S_{0}^{{ }^{\omega^{-1}} \rightarrow \tilde{R} S_{0}}$ (considered as elements of $\left.\operatorname{End}_{\tilde{R}}\left(\tilde{R} S_{0}\right)\right)$ may be chosen such that $t_{w^{\prime}} t_{w^{\prime}}=t_{w^{\prime} w^{\prime}}$ for all $w, w^{\prime} \in W$. In other words, in the language of stable Clifford theory (see e.g. [6]), $\operatorname{End}_{\tilde{R} H}\left(\tilde{R} S_{0}^{H}\right) \cong$ $\tilde{R} W(\cong \tilde{R}(H / L))$ canonically.

In this section we will give a presentation of $\operatorname{End}_{\tilde{R}_{G}}\left(\tilde{R} S^{G}\right)$. For $\tilde{R}=K$ this has been done by R. B. Howlett and G. I. Lehrer in [12] in a more general situation. They proved in particular that End ${ }_{K G}\left(K S^{G}\right)$ is the Hecke algebra $K\left[W_{s}\right.$ ] (later we will give a concrete description of this algebra). This will be generalized to arbitrary $\tilde{R} \in\{\bar{R}, R, K\}$. But first we have to describe $S_{P}^{G}$.

Consider $W_{L}$, the Weyl group of $L$. Note that $W_{L} \leq W_{0}$. By [2, Chapter IV, 2.5] there exists a bijection between the double cosets of $W_{L}$ in $W_{0}$ and the double cosets of $P$ in $G$ given by $W_{L} w W_{L} \rightarrow P w P\left(w \in W_{0}\right)$.

\subsection{Lemma. Let $w, w^{\prime} \in W$. Then $w^{\prime} \in P w P$ if and only if $w=w^{\prime}$.}

Proof. By the above it is enough to prove $w^{\prime} \in W_{L} w W_{L}$, if and only if $w=w^{\prime}$. Considering $W$ as a symmetric group. $W_{L}$ becomes a Young subgroup of $W$ and the lemma follows from the well-known description of the double cosets of Young subgroups of symmetric groups.

Next we want to decompose the double coset $P w P(w \in W)$ into right cosets of $P$ in $G$. So let $V_{0} \leq G$ be the subgroup of $G$ of all unipotent, lower triangular matrices, and set, for $w \in W_{0}, U_{w}^{-}=V_{0}^{w^{-1}} \cap U_{0}$ and $U_{w}^{+}=U_{0} \cap U_{0}^{w}$. It is well known that $U_{0}=U_{w}^{+} U_{w}^{-}$and $U_{w}^{+} \cap U_{w}^{-}=(1)$. Let $B \leq G$ be the group of all upper triangular matrices in $G$ (Borel subgroup of $G$ ). Then every element of $B w B \subset G$ may be uniquely written as $g w u$ with $g \in B$ and $u \in U_{u}^{-}$(the Bruhat decomposition). So $B w B$ is the disjoint union $\dot{U}_{u \in U_{n}^{-}} B w u$. Now let in particular $w \in W_{s}$. Then $U_{L}=$ $L \cap U_{0}$ is contained in $U_{w^{+}}^{+}$, because $w$ permutes the factors of $U_{L}$. Of course $U_{0}=U_{I} \cdot U$, hence $U_{w^{-}} \subset U$. Therefore

$$
P w P=P w L U=P w U=P w U_{w}^{-}=\bigcup_{u \in U_{w^{-}}^{-}} P w u .
$$

Let $u_{1}, u_{2} \in U_{w}^{-}$and assume $w u_{1} \in P w u_{2}$. Then $w u_{1}=u_{3} x w u_{2}$ for some $x \in L$ and $u_{3} \in U$. By the Bruhat decomposition for $L$ there exists $g \in B \cap L, w_{1} \in W_{L}$ and 
$u_{4} \in U_{L} \cap U_{w_{1}}^{-}$such that $x=g w_{1} u_{4}$. Now $u_{4} \in U_{L} \subset U_{w}^{+}$, so $w u_{1}=u_{3} g w_{1} w u_{5} u_{2}$ with $u_{5}=u_{4}^{w} \in B$, hence $w u_{1} \in B w_{1} w B$ forcing $w_{1}=1$. Thus $x \in B$, and again by the Bruhat decomposition $u_{3} x=1$ and $u_{1}=u_{2}$. We have shown

3.2. Lemma. $P w P=\dot{U}_{u \in U_{w}^{-}} P w u$ (disjoint union) $\left(w \in W_{s}\right)$.

We still need

3.3. Lemma. Let $V$ be a free finitely generated $R$-module with basis $\left\{v_{i} \mid i \in I\right\}$ for some index set I. Let $J_{t}(t \in \mathscr{T})$ be a family of pairwise disjoint subsets of $I$, $x_{t}=\sum_{i \in J_{t}} v_{i}$. Then the sum $\sum_{t \in \mathscr{T}} x_{t} R$ is direct and has an $R$-complement in $V$.

Proof. Obvious.

Remember that $f=|U|^{-1} \sum_{u \in U} u$. Let $\tilde{R} \in\{\bar{R}, R, K\}$. Then

3.4. LeMma. $\tilde{R} S_{P}^{G}=\sum_{w \in W}^{\oplus}(\tilde{R} S \otimes 1) f w f \oplus \tilde{R} S^{G}(1-e)$.

Proof. $\tilde{R} S^{G}=\sum \tilde{R} S \otimes g$ as a $\tilde{R}$-space, where $g$ runs through a system of right coset representatives of $P$ in $G$, which we may choose such that it contains $\left\{w u \mid w \in W, u \in U_{w}^{-}\right\}$by 3.2. Therefore $\sum_{w \in W}(\tilde{R} S \otimes 1) f w f+\tilde{R} S^{G}(1-e) \leq \tilde{R} S^{G}$. Note that $f=f_{w}^{+} f_{w}^{-} \quad(w \in W)$ with $f_{w}^{+}=\left|U_{w}^{+} \cap U\right|^{-1} \sum_{u \in U \cap U_{w}^{+}} u$ and $f_{w}^{-}=$ $\left|U_{u^{-}}^{-}\right|^{-1} \sum_{u \in U_{n}^{-}} u$, because $U=\left(U_{w}^{+} \cap U\right) U_{w}^{-}$. Furthermore $u^{w^{-1}} \in U^{w} \cap U_{w}^{+}$for $u \in$ $U \cap U_{w^{\prime}}^{+}$, hence

$$
(S \otimes 1) f w f=S \otimes w f=S \otimes w f_{w}^{+} f_{w}^{-}=S \otimes w f_{w}^{-}=S \otimes \widehat{w U_{w}^{-}},
$$


that the sum $\sum_{w \in W}(S \otimes 1) f w f$ is direct and has an $R$-module complement in $S^{G}$. Furthermore $(S \otimes 1) f w f \cong S^{w^{-1}} \cong S$ as an $L$-module, because $g \in L$ commutes with $f$, and $S$ is trivial as a $U$-module, hence $(S \otimes 1) f w f \cong S$ as a $P$-module. Of course $\sum_{w \in W}(S \otimes 1) f w f \leq S^{G} \cdot e$, thus the sum $X=\sum_{w \in W}(S \otimes 1) f w f+S^{G}(1-e) \leq S^{G}$ is direct, and, by the above, has an $R$-complement in $S^{G}$. Of courses this is a decomposition of $X$ as a $P$-module. From 2.4 and the Frobenius reciprocity it follows that $K S^{G}{ }_{P}=|W| \cdot K S \oplus V$, where the constituents of $V$ are not contained in $b$, i.e. $V=K S^{G}(1-e)$. Thus $X$ is an $R G$-lattice in $K S^{G}$, hence, counting $R$-rank, $X=S^{G}$. Tensoring by $\tilde{R}$ we get the desired result.

Recall the definition of $t_{w} \in \operatorname{End}_{R}(S)(w \in W)$. Define $A_{w} \in \operatorname{Hom}_{R P}\left(S, S^{G}\right)$ by $A_{w}(s)=t_{w}(s) \otimes f w f$. Then, by 3.4, $\left\{A_{w} \mid w \in W\right\}$ is an $R$-basis of $\operatorname{Hom}_{R P}\left(S, S^{G}\right)$. By the Nakayama relations every $A_{w}, w \in W$, induces an element $B_{w} \in \operatorname{End}_{R G}\left(S^{G}\right)$ defined by $B_{w}(s \otimes g)=A_{w}(s) g$ for all $g \in G$. Note that for $w=1 \in W, B_{w}=B_{1}$ $=1 \in \operatorname{End}_{R G}\left(S^{G}\right)$. Consider $K S^{G}$ and extend $B_{w}$ to an endomorphism of $K S^{G}$ also denoted by $B_{w}\left(=1 \otimes B_{w}\right)$. It can easily be seen that our definition of $B_{w}$ coincides with the one given in $[12,3.8]$.

Remember that $C_{G}(s)=\prod \mathrm{GL}_{m_{\Lambda}(s)}\left(q^{\operatorname{deg} \Lambda}\right) \leq G, W=W_{C_{G}(s)}$. Let $W=\Pi W(\Lambda)$ be the corresponding decomposition of $W$, where we may consider $W(\Lambda)$ as the symmetric group $S_{m_{\Lambda}(s)}$ permuting the natural basis of the vector space $\left(\mathrm{GF}\left(q^{\operatorname{deg} \Lambda}\right)\right)^{m_{\Lambda}(s)}$ over $\operatorname{GF}\left(q^{\operatorname{deg} \Lambda}\right)$. Let $\mathscr{T}(\Lambda)$ be the set of basic transpositions in $W(\Lambda)$ (that is the permutation matrices corresponding to the transpositions of the 
form $(i, i+1)$ in $\left.S_{m_{1}(s)}, 1 \leq i \leq m_{\Lambda}(s)-1\right)$. Then $\mathscr{T}=\bigcup \mathscr{T}(\Lambda)$ generates $W$, where the union has to be taken over all $\Lambda \in \mathscr{F}$. Furthermore, for $w \in W$, let $\operatorname{lt}(w)$ denote as usual the number of factors in a reduced (i.e. minimal) expression of $w$ as product of basic transpositions, and note that $\operatorname{lt}(w)$ is uniquely determined.

The following lemma has been proved by R. B. Howlett and G. I. Lehrer in [12] considering $B_{w}(w \in W)$ as element of $\operatorname{End}_{K G}\left(K S^{G}\right)$ in a more general context. We will give here a more simple proof of it (corresponding to the more simple situation), since we will need parts of the proof later on.

3.5. Lemma. Let $\Lambda \in \mathscr{F}, \operatorname{deg} \Lambda=d, v \in \mathscr{T}(\Lambda)$ and $w \in W$. Then

$$
B_{v^{\prime}} \cdot B_{w^{\prime}}= \begin{cases}B_{v^{\prime} w^{\prime}} & \text { if } \operatorname{lt}(v w)>\operatorname{lt}(w), \\ q^{-d^{2} B_{l^{\prime} w^{\prime}}+\beta B_{w^{\prime}}} & \text { otherwise, }\end{cases}
$$

with $\beta=\varepsilon q^{d(d-1) / 2-d^{2}}\left(q^{d}-1\right)$, where $\varepsilon=1$ if the order of $(\Lambda) \in L(\Lambda)$ is odd and $\varepsilon=-1$ otherwise.

Proof. It is enough to calculate $B_{v} B_{w}(m \otimes 1) \in S^{G}$ for $m \in S$. Now

$$
B_{l} B_{w}(m \otimes 1)=t_{t} t_{w}(m) \otimes f v f f w f=t_{v^{\prime}}(m) \otimes f v f w f .
$$

Recall from 3.4 the definition of $f_{v}^{-}, f_{v}^{+}$and note that $f=f_{v}^{+} f_{v}^{-}$. Thus

$$
f v f w f=f v f_{v^{-}}^{-} w f=\left|U_{v^{-}}\right|^{-1} \sum_{u \in U_{r^{-}}^{-}} f v u w f .
$$

As an easy consequence of $[3,2.5 .8]$ we get $\mathrm{lt}_{0}(v w)=\mathrm{lt}_{0}(v)+\mathrm{lt}_{0}(w)$ if $\operatorname{lt}(v w)>$ $\operatorname{lt}(w)$, i.e. $\operatorname{lt}(v w)=\operatorname{lt}(w)+1$, where $\operatorname{lt}_{0}: W_{0} \rightarrow \mathbf{N}$ denotes the length function on $W_{0}$, the Weyl group of $G$. Thus $[3,7.2 .1]$ implies $U_{v}^{-} \leq U_{w}^{+}$, hence $f v f_{v}^{-} w f=f v w f$ and $B_{r} B_{n}(m \otimes 1)=t_{v w}(m) \otimes f v w f=B_{v^{\prime} w}(m \otimes 1)$ if $\operatorname{lt}(v w)>\operatorname{lt}(w)$, as desired. So we may assume that $\operatorname{lt}(v w) \leq \operatorname{lt}(w)$, i.e. $\operatorname{lt}(v w)=\operatorname{lt}(w)-1$. By the above, using induction on the length of $w$, we may assume that $w=v$.

Now let $D \subset W_{0}$ be a set of representatives of double cosets of $P$ containing $W$ (3.1). Then $S_{P}^{G}=\sum_{x \in D}^{\oplus} S_{x}$, with $S_{x}=(S \otimes x)_{x^{-1} P x \cap P}{ }^{P}$ by Mackey's decomposition theorem. For $x \in D$ set $a_{x}=\sum_{u \in U_{x}^{-}, v u v \in P_{x} P} f v u v f$. Then $m \otimes a_{x} \in S_{x}$, in particular, $\sum_{x \in D, x \notin W} m \otimes a_{x} \in S^{G}(1-e)$, since $S_{x} \subset S^{G}(1-e)$ for $x \in D, x \notin W$ by 3.4. Furthermore, $\sum_{x \in w^{m}} \otimes a_{x} \in S^{G} \cdot e$, again by 3.4. Now

$$
B_{r}^{2}(m \otimes 1)=\left|U_{v}^{-}\right|^{-1} \sum_{x \in D} m \otimes a_{x}=\left|U_{v}^{-}\right|^{-1} \sum_{x \in W} m \otimes a_{x}+\left|U_{v}^{-1}\right|^{-1} \sum_{x \in D, x \in W} m \otimes a_{x} .
$$

Since $B_{v^{\prime}}^{2}\left(S^{G} e\right)=\left(B_{v}^{2}\left(S^{G}\right)\right) e \leq S^{G} e$ we conclude that

$$
\sum_{x \in D, x \notin W} m \otimes a_{x} \in S^{G} e \cap S^{G}(1-e)=(0) .
$$

In particular, $a=\sum_{x \in D, x \notin W} a_{x}$ annihilates the $P$-submodule $S^{G} e$ of $S^{G}$ and

$$
B_{v}^{2}(m \otimes 1)=\left|U_{v}^{-}\right|^{-1} \sum_{x \in W} m \otimes a_{x} .
$$

Thus assume that $v u v \in P x P$ with $x \in W$. Let $v=v_{1} \cdots v_{k}$ be a reduced expression for $v$ as element of $W_{0}$. By $[3,8.1 .5] v u v \in B y B \subseteq P y P, y \in W$, where a reduced expression of $y$ as element of $W_{0}$ involves only basic transpositions $v_{i} \in W_{0}$, 
$1 \leq i \leq k$. By [2, Chapter IV, 2.5] $W_{L} x W_{L}=W_{L} y W_{L}$, and we conclude $x=v$ or $x=1$. Now

$$
v u v=\left(\begin{array}{llllll}
E . & & & & & \\
& \cdot & E & 0 & & \\
& A & E & & \\
& & & & \cdot E
\end{array}\right)
$$

for a certain $(d \times d)$-matrix $A$, where $E$ denotes the $(d \times d)$-identity matrix. A quick calculation shows that $v u v \in P v P$ if and only if $A$ is invertible, and then $v u v=u^{\prime} s_{A} v u^{\prime}$ with

$$
u^{\prime}=\left(\begin{array}{cccccc}
E & & & & & \\
& \ddots & & & & \\
& & E & A^{-1} & & \\
& & 0 & E & \ddots & \\
& & & & & E
\end{array}\right)
$$

and

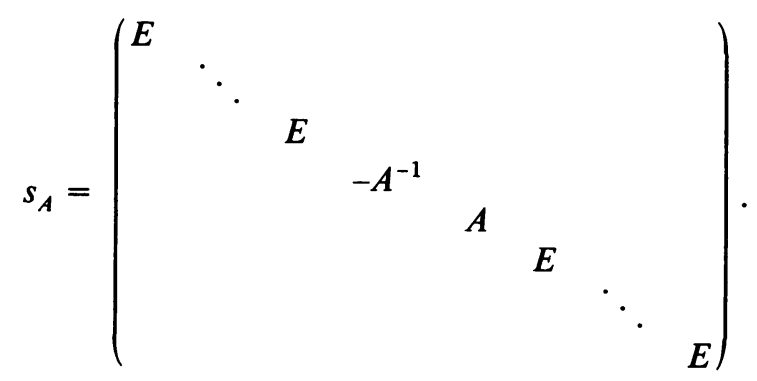

Of course $v u v \in P \cdot 1 \cdot P=P$ if and only if $A=0$, i.e. $u=1$.

Summarizing we get

$$
B_{v}^{2}(m \otimes 1)=t_{v^{2}}(m) \otimes f v f v f=\left|U_{v}^{-}\right|^{-1}(m \otimes 1)+\left|U_{v}^{-}\right|^{-1} t \otimes f v f
$$

with $t=\sum_{A \in \mathrm{GL}_{d}(q)} m s_{A}$.

For an arbitrary finite group $G$ and an irreducible representation $T: G \rightarrow \mathrm{GL}(V)$ over $\mathbf{C}$, the well-known formula

$$
\sum_{g \in G} t_{i j}\left(g^{-1}\right) t_{k l}(g)=\delta_{j k} \delta_{i l} \frac{|G|}{\operatorname{dim} V}
$$

where $\mathrm{GL}(V) \ni\left(t_{i j}(g)\right)=T(g)$ for $g \in G$, implies immediately that

$$
\begin{aligned}
\sum_{g \in G} e_{i} T\left(g^{-1}\right) \otimes_{\mathrm{C}} e_{k} T(g) & =\sum_{j, s} e_{j} \otimes_{\mathrm{C}} e_{s}\left(\sum_{g \in G} t_{i j}\left(g^{-1}\right) t_{k s}(g)\right) \\
& =\frac{|G|}{\operatorname{dim} V} \sum_{j, s} e_{j} \otimes_{\mathrm{C}} e_{s} \delta_{j k} \delta_{i s}=\frac{|G|}{\operatorname{dim} V} e_{k} \otimes_{\mathrm{C}} e_{i}
\end{aligned}
$$

for all elements $e_{i}, e_{k}$ of the natural basis of $V$, hence

$$
\sum_{g \in G} v_{1} T\left(g^{-1}\right) \otimes_{\mathrm{C}} v_{2} T(g)=\frac{|G|}{\operatorname{dim} V} v_{2} \otimes_{\mathrm{C}} v_{1}
$$


for all $v_{1}, v_{2} \in V .^{2}$ Applying this to our situation, replacing $\mathbf{C}$ by $K$ and $V$ by the irreducible $R L(\Lambda)$-lattice $S(\Lambda)$, we easily get $t=\varepsilon|L(\Lambda)| t_{v}(m) / \operatorname{dim} S(\Lambda)$, observing that $m(\Lambda) \cdot A=\varepsilon m(\Lambda)$ for $A=-E$ by $[15,6.8]$. By $[15,6.21] \operatorname{dim} S(\Lambda)=$ $|L(\Lambda)| /\left|U_{L(\Lambda)}\right||T(\Lambda)|$, where $U_{L(\Lambda)}$ is the subgroup of all upper triangular unipotent matrices of $L(\Lambda)$. Now $\left|U_{v^{-}}\right|=q^{d^{2}},\left|U_{L(\Lambda)}\right|=q^{d(d-1) / 2}$ and $|T(\Lambda)|=q^{d}-1$. Thus

$$
\begin{aligned}
B_{v}^{2}(m \otimes 1) & =q^{-d^{2}}(m \otimes 1)+\varepsilon q^{d(d-1) / 2-d^{2}}\left(q^{d}-1\right) t_{v}(m) \otimes f v f \\
& =q^{-d^{2}}(m \otimes 1)+\beta B_{v}(m \otimes 1)
\end{aligned}
$$

for all $m \in S$, hence $B_{v}^{2}=q^{-d^{2}}+\beta B_{v}$ as desired.

As an immediate consequence from the proof above we get

Corollary. Let $w_{1}, w_{2} \in W$. Then $f w_{1} f w_{2} f=\sum_{w \in W} r_{w} f w f+$ a for certain $r_{w} \in R L$, where $a \in R G$ annhilates $S^{G} \cdot e \subseteq S^{G}$.

Now, for $\Lambda \in \mathscr{F}, v \in \mathscr{T}(\Lambda), d=\operatorname{deg} \Lambda$ and $w \in W$ define $T_{v}=\varepsilon q^{d(d+1) / 2} B_{v} \in$ End $_{R G}\left(S^{G}\right)$ and $T_{w}=T_{v_{1}} \cdots T_{v_{h}}$ if $w=v_{1} \cdots v_{k}$ is a reduced expression for $w$. By the above $T_{w}=q^{m} B_{w}$ for a certain $m \in \mathbf{N}$. Of course, by $3.4,\left\{T_{w} \mid w \in W\right\}$ is a basis of $\operatorname{End}_{\tilde{R} G}\left(\tilde{R} S^{G}\right)$ for $\tilde{R} \in\{\bar{R}, R, K\}$, denoting $1 \otimes T_{w} \in \tilde{R} \otimes_{R} \operatorname{End}_{R G}\left(S^{G}\right)$ again by $T_{n}$. Furthermore, by 3.5, $T_{v} \cdot T_{w}=T_{v w}$, if $\operatorname{lt}(v w)>\operatorname{lt}(w)$ and $T_{v} T_{w}=q^{d} T_{v w}+$ $\left(q^{d}-1\right) T_{w}$ otherwise, where $v, w \in W$ as in 3.5. For an arbitrary commutative ring $\tilde{R}$ (with 1) let $\tilde{R}[W]$ be the $\tilde{R}$-free $\tilde{R}$-algebra with basis $\left\{T_{w} \mid w \in W\right\}$, where the multiplication is induced by the relations above. We will see later that $\tilde{R}[W]$ is an associative $\tilde{R}$-algebra for every choice of $\tilde{R}$. Of course $\bar{R}[W] \cong \bar{R} \otimes_{R} R[W]$ and $K[W] \cong K \otimes_{R} R[W]$ canonically, thus we have shown, generalizing [12] partially:

3.6. THEOREM. Let $s \in G$ be semisimple satisfying 2.4. Then $\operatorname{End}_{\tilde{R} G}\left(\tilde{R} S^{G}\right)=\tilde{R}[W]$ $=\tilde{R} \cdot \operatorname{End}_{R G}\left(S^{G}\right)$ for every choice of $\tilde{R} \in\{\bar{R}, R, K\}$.

For the rest of this paper let $A$ be the subalgebra of $R G$ generated by $\{f R P f, f w f \mid w \in W\}$. Note that $f$ is the identity of $A$. Furthermore, $e A=A e$, thus $M \cdot e \leq M_{A}$ for an arbitrary $R G$-module $M$, where $M_{A}$ denotes the restriction of $M$ to the subalgebra $A$ of $R G$. In particular,

$$
S^{G} \cdot e=\sum_{n \in W} S \otimes f w f=(S \otimes 1) A \leq S_{A}^{G} .
$$

Let $I$ be the annihilator ideal of $S^{G} \cdot e$ in $A$, and $\pi: A \rightarrow A / I$ the natural epimorphism. Then $\mathscr{A}=A / I$ is $R$-free, being isomorphic to a subalgebra of the $R$-free $R$-algebra $\operatorname{End}_{R}\left(S^{G} \cdot e\right)$. From 3.4 and the corollary to 3.5 we conclude immediately that $\mathscr{A}=\sum_{w \in W}^{\oplus} \pi(f R P f) \pi(f w f)=\sum_{w \in W}^{\oplus} \pi(f w f) \pi(f R P f)$, i.e. $\mathscr{A}$ is free as a $\pi(f R P f)$-module with basis $\{\pi(f w f) \mid w \in W\}$ (on both sides). Note that $\pi(f R P f) \cong R L / I_{0}$ canonically, where $I_{0}$ denotes the annihilator of $S$ in $R L$. Of course, $K I$ is the annihilator of $K S^{G} e$ in $K A$, and $K \mathscr{A} \cong K A / K I$ canonically. Let $I_{1}$ be the annihilator of $\bar{R} S^{G} e$ in $\bar{R} A$, and $\pi_{1}: \bar{R} A \rightarrow \bar{R} A / I_{1}=\mathscr{A}_{1}$ the natural epimorphism. Because $A, I$ and $A / I=\mathscr{A}$ are $R$-free, we may consider $\bar{R} I$ as an ideal of $\bar{R} A$ canonically, and of course $\bar{R} I \subset I_{1}$. Thus there exists an epimorphism from $\bar{R} A / \bar{R} I$

\footnotetext{
2I am indebted to R. Knörr who showed me the proof of this general result.
} 
onto $\mathscr{A}_{1}$. Now 3.5 holds analogously if we replace $S$ by $\bar{R} S$ and $R G$ by $\bar{R} G$, by 3.4 . Consequently $\mathscr{A}_{1}=\sum_{w \in W}^{\oplus} \pi_{1}(\overline{f R} P f) \pi_{1}(f w f)$, and $\pi_{1}(f \bar{R} P f) \cong \bar{R} L / I_{2}$, where $I_{2}$ denotes the annihilator of $\bar{R} S$ in $\bar{R} L$. We again get an epimorphism from $\bar{R} L / \bar{R} I_{0}$ onto $\bar{R} L / I_{2}$. Since $\bar{R} S$ and $K S$ are irreducible, we get, counting dimensions, $\bar{R} L / \bar{R} I_{0} \cong$ $\bar{R} L / I_{2}$, i.e. $\bar{R} I_{0}=I_{2}$. This implies $\bar{R} A / \bar{R} I \cong \mathscr{A}_{1}$. Of course $\bar{R} A / \bar{R} I \cong \bar{R} \mathscr{A}$. Thus $\tilde{R} \mathscr{A}$ is isomorphic to $\tilde{R} A$ modulo the annihilator of $\tilde{R} S^{G} e$ in $\tilde{R} A$ for every choice of $\tilde{R} \in\{\bar{R}, R, K\}$ canonically.

3.4 and 3.5 immediately imply that $\tilde{R} S^{G} e=(S \otimes 1) \tilde{R} \mathscr{A} \cong \tilde{R} S \otimes_{\pi(f \tilde{R} P f)} \tilde{R} \mathscr{A}$, the induced module. Let $\tau \in \operatorname{End}_{\tilde{R} G}\left(\tilde{R} S^{G}\right)$. Since $\tau\left(\tilde{R} S^{G} e\right) \leq \tilde{R} S^{G} e$, the restriction $\tilde{\tau}$ of $\tau$ to $\tilde{R} S^{G} e$ defines an endomorphism of $\tilde{R} S^{G} e$ as an $\tilde{R} \mathscr{A}$-module. By adjointness of induction and restriction (Frobenius reciprocity), $\operatorname{End}_{\tilde{R} \mathscr{A}}\left(\tilde{R} S^{G} e\right) \cong$ $\operatorname{Hom}_{\pi(f \tilde{R} P f)}\left(\tilde{R} S, \tilde{R} S^{G} e\right)$. So 3.4 implies immediately that $\left\{\tilde{T}_{w} \mid w \in W\right\}$ is a basis of $\operatorname{End}_{\tilde{R} \mathscr{A}}\left(\tilde{R} S^{G} e\right)$, and we may identify $\operatorname{End}_{\tilde{R} G}\left(\tilde{R} S^{G}\right)$ and $\operatorname{End}_{\tilde{R} \mathscr{A}}\left(\tilde{R} S^{G} e\right)$. Furthermore, $\tilde{R}[W] \cong \tilde{R} \mathscr{A} / \tilde{R} \Omega_{p}$, where $\Omega_{p}$ is the ideal of $\mathscr{A}$ generated by $\{\pi(f-f g f) \mid g \in$ $P\}$. The next lemma tells us that we may argue as in stable Clifford theory, regarding $\tilde{R} \mathscr{A}$ as a kind of semidirect product of $\pi(f \tilde{R} P f)$ with $\tilde{R}[W]$, and as an "intertial algebra" of $\tilde{R} S$ in $\tilde{R} G$.

Let $\bmod (\tilde{R}[W] / \tilde{R})$ be the category of all finitely generated $\tilde{R}$-free $\tilde{R}[W]$-modules and $\bmod (\tilde{R} \mathscr{A} / \tilde{R} S)$ the category of all finitely generated $\tilde{R} S$-homogeneous $\tilde{R} \mathscr{A}$-modules, where we call an $\tilde{R} \mathscr{A}$-module $\tilde{R} S$-homogeneous if its restriction to $\pi(f \tilde{R} P f)$ is isomorphic to a direct sum of copies of $\tilde{R} S$.

3.7. Lemma. The functors ? $\otimes_{\tilde{R}[W]}(S \otimes 1) \tilde{R} \mathscr{A}$ and $\operatorname{Hom}_{\tilde{R} \mathscr{A}}((S \otimes 1) \tilde{R} \mathscr{A}, ?)$ form an equivalence between $\bmod (\tilde{R}[W] / \tilde{R})$ and $\bmod (\tilde{R} \mathscr{A} / \tilde{R} S)$.

Proof. Obviously ? $\otimes_{\tilde{R}[W]}(S \otimes 1) \tilde{R} \mathscr{A}$ is a functor from $\bmod (\tilde{R}[W] / \tilde{R})$ to $\bmod (\tilde{R} \mathscr{A} / \tilde{R} S)$ and $\operatorname{Hom}_{\tilde{R} \mathscr{A}}((S \otimes 1) \tilde{R} \mathscr{A}, ?) \operatorname{from} \bmod (\tilde{R} \mathscr{A} / \tilde{R} S)$ to $\bmod (\tilde{R}[W] / \tilde{R})$. The Nakayama relations show that $\operatorname{Hom}_{\tilde{R} \mathscr{A}}((S \otimes 1) \tilde{R} \mathscr{A}, X) \cong \operatorname{Hom}_{f \tilde{R} P f}(\tilde{R} S, X)$ as $\tilde{R}$-modules by restriction of $a:(S \otimes 1) \tilde{R} \mathscr{A} \rightarrow X$ to $\tilde{R} S=\tilde{R} S \otimes 1$ for $X \in$ $\bmod (\tilde{R} \mathscr{A} / \tilde{R} S)$. Note also that

$$
B \otimes_{\tilde{R}[W]}((S \otimes 1) \tilde{R} \mathscr{A}) \cong B \otimes_{\tilde{R}[W]}\left(\tilde{R}[W] \otimes_{\tilde{R}} \tilde{R} S\right) \cong B \otimes_{\tilde{R}} \tilde{R} S
$$

as $f \tilde{R} P f$-modules for $B \in \bmod (\tilde{R}[W] / \tilde{R})$.

Now define

$$
\varepsilon: \operatorname{Hom}_{\tilde{R} \mathscr{A}}((S \otimes 1) \tilde{R} \mathscr{A}, X) \otimes_{\tilde{R}[W]}(S \otimes 1) \tilde{R} \mathscr{A} \rightarrow X
$$

as the homomorphism induced by

$$
\varepsilon(a \otimes m)=a(m)
$$

for $a \in \operatorname{Hom}_{\tilde{R} \mathscr{A}}((S \otimes 1) \tilde{R} \mathscr{A}, X)$ and $m \in(S \otimes 1) \tilde{R} \mathscr{A}$, and

$$
\gamma: B \rightarrow \operatorname{Hom}_{\tilde{R} \mathscr{A}}\left((S \otimes 1) \tilde{R} \mathscr{A}, B \otimes_{\tilde{R}[W]}(S \otimes 1) \tilde{R} \mathscr{A}\right)
$$

by $\gamma(b)=a_{b} \in \operatorname{Hom}_{\tilde{R} \mathscr{A}}\left((S \otimes 1) \tilde{R} \mathscr{A}, \quad B \otimes_{\tilde{R}[W]}(S \otimes 1) \tilde{R} \mathscr{A}\right)$ for $b \in B$, where $a_{b}(x)=b \otimes x$ for $x \in \tilde{R} S \otimes 1$. 
Obviously $\varepsilon$ is natural in $X$ and $\gamma$ in $B$. Now as in stable Clifford theory (see e.g. [6]) it can easily be seen, by the remarks made above, that $\varepsilon$ and $\gamma$ are isomorphisms. This proves the lemma.

In the following we identify $\bmod (\tilde{R} \mathscr{A} / \tilde{R} S)$ with the full subcategory of $\bmod (\tilde{R} A / \tilde{R} S)$ of all finitely generated $\tilde{R} S$-homogeneous $\tilde{R} A$-modules, which are annihilated by $\tilde{R} I$, where $\bmod (\tilde{R} A / \tilde{R} S)$ denotes the category of all finitely generated $\tilde{R} S$-homogeneous $\tilde{R} A$-modules. Note that simple objects of $\bmod (\tilde{R} \mathscr{A} / R S)$ are irreducible $\tilde{R} A$-modules.

3.8. Corollary. $I \rightarrow I(S \otimes 1) \tilde{R} A$ defines an isomorphism between the lattice of all $\tilde{R}$-free right ideals $I$ of $\tilde{R}[W]$ and the lattice of all $\tilde{R} S$-homogeneous submodules of $(S \otimes 1) \tilde{R} A$.

4. Theorem 3.6 and Corollary 1.6 particularly imply that $K W \cong K[W]$. In fact this is a well-known theorem, not only for Weyl groups of type $A_{n}$, but also for arbitrary Coxeter groups, if $K$ contains $\mathbf{Q}\left[q^{1 / 2}, q^{-1 / 2}\right]$. In our case $\left(W=W_{s}\right)$ it is true for $K=\mathbf{Q}$. The proof of the general theorem uses the fact that separable algebras with the same numerical invariants (the degrees of the irreducible characters) have to be isomorphic by Wedderburn's theorem.

In the following we will identify $\mathbf{Q}[W]$ and $\mathbf{Q} W$ by a certain isomorphism, which we will extend to an isomorphism between $K[W]$ and $K W$. So we will find in $K[W]$ two $R$-orders, one generated by the basis $\left\{T_{w} \mid w \in W\right\}$, the other by the basis $\{w \mid w \in W\} \subset K W=K[W]$, namely $R[W]$ and $R W$ respectively. These are not isomorphic in general, in fact, choosing $s=1$ in $G=\mathrm{GL}_{2}(5)$ and $r=3$, then $W=S_{2}$ and $\bar{R} S_{2}=\bar{R} \otimes_{R} R S_{2}$ is semisimple, whereas $\bar{R}\left[S_{2}\right]=\bar{R} \otimes R\left[S_{2}\right]$ has a one-dimensional nilpotent ideal, namely $\left\{a+a T_{w} \mid a \in \bar{R}\right\}$, where $w$ is the unique element of order 2 in $S_{2}$. In particular, $R S_{2}$ and $R\left[S_{2}\right]$ cannot be isomorphic in this case.

The proof of the theorem mentioned above uses the generic algebra. We will introduce it here only for Weyl groups of type $A_{n}$ (i.e. symmetric groups) using $K$ instead of $\mathbf{Q}\left[q^{1 / 2}, q^{1 / 2}\right]$. For the general case, more details and proofs of the following claims, the reader is referred to $[1,2,4,13]$.

So let $\left\{W_{i} \mid i \in I\right\}$ be a family of symmetric groups $W_{i}$ over a finite index set $I$, and let $\mathbf{d}=\left\{d_{i} \mid i \in I\right\}$ be a family of positive integers. Let $K\left[u, u^{-1}\right]$ be the ring of Laurent polynomials over $K$ in the indeterminant $u, K(u)$ the quotient field of $K\left[u, u^{-1}\right]$. Let $W=\prod_{i \in I} W_{i}$. Then the generic $K$-algebra $\mathscr{G}[W, \mathbf{d}]$ of $W$ over $K$ with respect to d is defined as follows: $\mathscr{G}[W, \mathrm{~d}]$ is a free $K\left[u, u^{-1}\right]$-module with basis $\left\{T_{n} \mid w \in W\right\}$, where the multiplication in $\mathscr{G}[W, \mathbf{d}]$ is given by

$$
T_{v} T_{w}=\left\{\begin{array}{l}
T_{v w} \quad \text { if } \operatorname{lt}(v w)=1 \mathrm{t}(w)+1, \\
u^{d^{d}} T_{v^{\prime} w}+\left(u^{d_{i}}-1\right) T_{w} \text { otherwise }
\end{array}\right.
$$

for $w \in W$ and $v \in W_{i} \subseteq W$, where $v$ is a basic transposition. Let $h_{\infty}: K\left[u, u^{-1}\right] \rightarrow$ $K(u)$ be the natural embedding, and, for $0 \neq k \in K$, let $h_{k}: K\left[u, u^{-1}\right] \rightarrow K$ be the 
homomorphism induced by $h_{k}(u)=k$. Consider $K$ and $K(u)$ as a $K\left[u, u^{-1}\right]$-module via $h_{k}$ and $h_{\infty}$ respectively, and set

$$
K[W, \mathbf{d}, k]=\mathscr{G}[W, \mathbf{d}] \otimes_{K\left[u, u^{-1}\right]} K
$$

and

$$
K[W, \mathbf{d}, \infty]=\mathscr{G}(W, \mathbf{d})=\mathscr{G}[W, \mathbf{d}] \otimes_{K\left[u, u^{-1}\right]} K(u)
$$

respectively.

$T_{n} \rightarrow T_{n} \otimes 1$ induces a $K$-algebra homomorphism from $\mathscr{G}[W, \mathbf{d}]$ into $K[W, \mathbf{d}, k]$ $(0 \neq k \in K \cup\{\infty\})$ which is also denoted by $h_{k}$ and is surjective for $k \in K$ and injective for $k=\infty$. For simplicity we will denote $T_{w} \otimes 1=h_{k}\left(T_{w}\right)$ by $T_{w}$ again.

We call $h_{k}(K[W, \mathbf{d}, k])$ the specialization of $\mathscr{G}[W, \mathbf{d}]$ with respect to $k$. Since $K[W, \mathbf{d}, q]$ ( $q$ an arbitrary prime power) is the endomorphism ring of an induced module (choosing $s=1$ in $G=\prod_{i \in I} \mathrm{GL}_{k_{i}}\left(q^{d_{i}}\right), k_{i} \in \mathbf{N}$, such that $W_{i} \cong S_{k_{i}}$ in 3.6), hence is in particular an associative algebra, it can easily be seen that $\mathscr{G}[\mathrm{W}, \mathbf{d}]$ is associative. This also remains true if we replace $K$ by an arbitrary commutative ring with 1 , because the multiplication of the $T_{w}$ 's $(w \in W)$ only involves Laurent polynomials in $u$ with integer coefficients.

Note that $K[W, \mathbf{d}, 1]=K W$, the usual group algebra of $W$ over $K$. Moreover, $T_{w}$ $(w \in W)$ is invertible in $\mathscr{G}[W, \mathbf{d}]$. In fact, if $v \in W_{i}$, lt $v=1$, then $T_{v}^{-1}=u^{-d_{i}} T_{v}+$ $\left(u^{-d_{i}}-1\right)$, and if $w=v_{1} \cdots v_{k}$ is a reduced representation of $w \in W$ as a product of basic transpositions, then $T_{w}^{-1}=T_{v_{k}}^{-1} \cdots T_{v_{1}}^{-1}$.

For $W=W_{s}$ we choose $I=\left\{\Lambda \in \mathscr{F} \mid m_{\Lambda}(s) \neq 0\right\}$ and $\mathbf{d}=\left\{d_{\Lambda}=\operatorname{deg} \Lambda \mid \Lambda \in I\right\}$. In particular, $K\left[W_{s}, \mathbf{d}, q\right]=K\left[W_{s}\right]$ and $K\left[W_{s}, \mathbf{d}, 1\right]=K W_{s}$, where $q$ is defined as in $\S 1$, and $K\left[W_{s}\right]$ as in $\S 3$. For simplicity we will write $\mathscr{G}\left[W_{s}\right]=\mathscr{G}[W]$ and $\mathscr{G}\left(W_{s}\right)=$ $\mathscr{G}(W)$ instead of $\mathscr{G}\left[W_{s}\right.$, d] $]$ and $\mathscr{G}\left(W_{s}\right.$, d) respectively, and we will formulate the following for $\mathscr{G}[W]$, although it is true in general, as we can see if we choose $s=1$ in $G$ as above. It can be shown that $\mathscr{G}(W)$ is semisimple. If $\chi$ is a character of $\mathscr{G}(W)$, then $\chi\left(T_{w}\right)$ is a polynomial in $u$ for all $w \in W$. Given a specialization $h_{k}: u \rightarrow k$ for some prime power $k$, then $\chi_{k}: K[W, \mathbf{d}, k] \rightarrow K$ defined by $\chi_{k}\left(T_{w} \otimes 1\right)$ $=h_{k}\left(\chi\left(T_{w}\right)\right)$ is a character of $K[W, \mathbf{d}, k]$. It turns out that $\chi_{k}$ is irreducible if and only if $\chi$ is irreducible. Of course $\chi_{k}(1)$ is independent of the choice of $k$. So we may identify $K[W]=K\left[W_{s}, \mathbf{d}, q\right]$ and $K W=K\left[W_{s}, \mathbf{d}, 1\right]$ by an isomorphism, say $\tau$, such that $\chi_{q}$ and $\chi_{1}$ define the same tharacter of $K[W]=K W$ for every irreducible character $\chi$ of $\mathscr{G}(W)$. Moreover, $\mathscr{G}(W)$ and $K[W, \mathbf{d}, k]$ have the same numerical invariants, and $\chi=\phi$ if and only if $\chi_{k}=\phi_{k}$ for two irreducible characters $\chi$ and $\phi$ of $\mathscr{G}(W)$. In particular, we get all irreducible characters of $K[W]=K W$ as specialized characters $\chi_{q}=\chi_{1}, \chi$ an irreducible character of $\mathscr{G}(W)$. Obviously $\chi_{q}=\chi_{1}$ for all characters $\chi$ of $\mathscr{G}(W)$.

Let $\mathscr{T}$ again be the set of basic transpositions of $W$, and $J \subset \mathscr{T}$. Then the subalgebra of $\mathscr{G}[W]$ generated by $\left\{T_{w} \mid w \in J\right\}$ is isomorphic to $\mathscr{G}\left[W_{J}\right]=\mathscr{G}\left[W_{J}, \mathbf{d}^{\prime}\right]$, where $W_{J}$ is the subgroup of $W$ generated by $J$ and $\mathbf{d}^{\prime}$ denotes the restriction of $\mathbf{d}$ to $\left\{\Lambda \in I \mid W_{J} \cap W(\Lambda) \neq(1)\right\}$. Similarly we define $\mathscr{G}\left(W_{J}\right)$, and in the following we always consider $\mathscr{G}\left[W_{J}\right]$ and $\mathscr{G}\left(W_{J}\right)$ as subalgebras of $\mathscr{G}[W]$ and $\mathscr{G}(W)$ respectively. 
If $0 \neq k \in K \cup\{\infty\}, h_{k}\left(K\left[W_{J}\right]\right)=K\left[W_{J}, \mathbf{d}^{\prime}, k\right] \subset K[W]$, i.e. $h_{k}$ restricted to $\mathscr{G}\left[W_{J}\right]$ is the specialization of $\mathscr{G}\left[W_{J}\right]$ with respect to $k$. In particular, $K\left[W_{J}\right]=K\left[W_{J}, \mathbf{d}, q\right]$ and $K W_{J}=K\left[W_{J}, \mathbf{d}^{\prime}, 1\right]$ may be considered as subalgebras of $K[W]=K W$. But we have to be careful here; an isomorphism between $K\left[W_{J}\right]$ and $K W_{J}$ cannot be extended to an isomorphism between $K[W]$ and $K W$ in general. In other words, $K\left[W_{J}\right]$ and $K W_{J}$ are different subalgebras of $K[W]=K W$ in general.

As above let $W_{J}$ be the subgroup of $W$ generated by $J$, and $R\left[W_{J}\right] \subset R[W]$ the $R$-order in $K\left[W_{J}\right]$ generated by $\left\{T_{w} \mid w \in W_{J}\right\}$. Note that $\bar{R}\left[W_{J}\right]=\bar{R} \otimes_{R} R\left[W_{J}\right]$. Let $D_{J}$ be a system of right coset representatives of $W_{J}$ in $W$. We may choose $D_{J}$ such that $\operatorname{lt}(w v)=\operatorname{lt}(w)+\operatorname{lt}(v)$ for all $w \in W_{J}, v \in D_{J}$, a so-called distinguished set of coset representatives (see e.g. [3, 2.5.8]).

4.1. Lemma. (i) $\mathscr{G}[W]$ and $\mathscr{G}(W)$ are free as $\mathscr{G}\left[W_{J}\right]$ - and $\mathscr{G}\left(W_{J}\right)$-left modules respectively with basis $\left\{T_{v} \mid v \in D_{J}\right\}$.

(ii) $\bar{R}\left[W_{J}\right] \subset \bar{R}[W]$, and $\bar{R}[W]$ is free as a $\tilde{R}\left[W_{J}\right]$-left module with basis $\left\{T_{v} \mid v \in D_{J}\right\}$ for every choice of $\tilde{R} \in\{\bar{R}, R, K\}$.

Proof. Choosing $D_{J}$ as a distinguished set of coset representatives, $\mathscr{G}[\mathrm{W}]=$ $\sum_{v^{\prime} \in D} \mathscr{G}\left[W_{J}\right] T_{v}$ as a $\mathscr{G}\left[W_{J}\right]$-left module. Counting $K\left[u, u^{-1}\right]$-rank, the sum must be direct. Now the lemma follows immediately.

Consider the trivial and the alternating character of $K W_{J}$. By the above these must be specializations of certain linear characters of $\mathscr{G}\left(W_{J}\right)$, which we will also call the trivial respectively alternating character of $\mathscr{G}\left(W_{J}\right)$. Being linear characters, the corresponding idempotents in $\mathscr{G}\left(W_{J}\right)$ are central and uniquely determined.

For $\Lambda \in \mathscr{F}$ and $J \subset \mathscr{T}$ let $W_{J}(\Lambda)=W_{J \cap \mathscr{T}(\Lambda)}=W_{J} \cap W(\Lambda)$, where $\mathscr{T}(\Lambda)=$ $W(\Lambda) \cap \mathscr{T}$. Let $y_{J}(\Lambda)=\sum_{w \in W_{J}(\Lambda)}(-1)^{\mathrm{lt} w} u^{-\operatorname{deg} \Lambda \mathrm{lt} w} T_{w}$. Define $y_{J}=\Pi y_{J}(\Lambda)$ and $x_{J}=\sum_{w \in W_{J}} T_{w}$.

4.2. Lemma. Let $J \subset \mathscr{T}$. Then there exist $\alpha, \beta \in K(u)$ such that $\alpha x_{J}$ and $\beta y_{J}$ are the unique idempotents in $\mathscr{G}\left(W_{J}\right)$ corresponding to the trivial and the alternating character of $\mathscr{G}\left(W_{J}\right)$ respectively.

PROof. Of course $x_{J}=\prod x_{J \cap \mathscr{T}(\Lambda)}$, hence we may assume that $W_{J}=W_{J}(\Lambda)$ for some $\Lambda \in \mathscr{F}$, i.e. $W_{J}$ has one nontrivial component, and the trivial and the alternating character are the only linear characters of $K W_{J}$. Obviously we may assume, too, that $\operatorname{deg} \Lambda=1$. So $\mathscr{G}\left(W_{J}\right)$ has just two different one-dimensional right ideals. Let $x=\sum_{w \in W_{J}} a_{w} T_{w} \in \mathscr{G}\left(W_{J}\right)$ such that $x \mathscr{G}\left(W_{J}\right)$ is one-dimensional over $K(u)$. This is equivalent to $x T_{v}=x \beta_{v}$ for all $v \in J$, where $\beta_{v} \in K(u)$, because $\mathscr{G}\left(W_{J}\right)$ is generated by $\left\{T_{v} \mid v \in J\right\}$ as algebra. Note that in particular $x \neq 0$. For $v \in J$ let $W_{v}=\left\{w \in W_{J} \mid \operatorname{lt}(v w)=\operatorname{lt}(w)+1\right\} ;$ then $W_{J}$ is the disjoint union of $W_{v}$ and $v W_{v}$, and $x=\sum_{w \in W_{v}}\left(a_{w} T_{w}+a_{v w} T_{v w}\right)$. Now $x$ is in the center of $\mathscr{G}\left(W_{J}\right)$, because it is a scalar multiple of some central idempotent. Consequently

$$
x T_{v}=T_{v} x=\sum_{w \in W_{v}} a_{v w} u T_{w}+\left(a_{w}+a_{v w}(u-1)\right) T_{v w} .
$$


So $x T_{v}=x \beta_{v}$, forces, for $w \in W_{v}$,

(i) $a_{i, w} u=a_{w} \beta_{v}$ and,

(ii) $a_{v^{\prime} w} \beta_{v^{\prime}}=a_{w}+a_{v w}(u-1)$.

Hence,

(iii) $a_{{ }^{\prime}}\left(\beta_{v}^{2}-\beta_{v}(u-1)-u\right)=0$.

(ii) implies $a_{w}=0$ if $a_{v w}=0$, hence, repeating the argument using a reduced expression for $w$ as the product of basic transpositions, $a_{1}=0$. Using (i) we conclude $a_{w^{\prime}}=0$ for all $w^{\prime} \in W_{J}$, forcing $x=0$, a contradiction. So $a_{w^{\prime}} \neq 0$ for all $w^{\prime} \in W_{J}$, and (iii) implies $\beta_{v}=u$ or $\beta_{v}=-1$.

Obviously the character $\chi$ afforded by $x \mathscr{G}\left(W_{J}\right)$ satisfies $\chi\left(T_{v}\right)=\beta_{v}$ for all $v \in J$. Therefore, using the specialization $u \rightarrow 1$, either $\beta_{v}=u$ for all $v \in \mathscr{T}$ (specializing to the trivial character of $W_{J}$ ) or $\beta_{v}=-1$ for all $v \in \mathscr{T}$ (specializing to the alternating character of $W_{J}$ ). Normalizing $a_{1}=1$ we get $x_{J}$ in the first and $y_{J}$ in the second case.

Identify $K[W]$ and $K W$ as above, and consider the subalgebras $K W_{J}$ and $K\left[W_{J}\right]$ $(J \subset \mathscr{T})$. Let $X_{J}^{(k)}=h_{k}\left(x_{J}\right) K W, Y_{J}^{(k)}=h_{k}\left(y_{J}\right) K W$ for $k=1$ or $q$.

4.3. LEMMA. $X_{J}^{(1)} \cong X_{J}^{(q)}$ and $Y_{J}^{(1)} \cong Y_{J}^{(q)}$ as $K W$-modules.

Proof. Let $D_{J}$ be a distinguished system of coset representatives of $W_{J}$ in $W$. Then $x_{J} \mathscr{G}[W] \cong x_{J} \mathscr{G}\left[W_{J}\right] \otimes_{\mathscr{G}\left[W_{J}\right]} \mathscr{G}[W]$ is a free $K\left[u, u^{-1}\right]$-module with basis $\left\{x_{J} \otimes T_{w} \mid w \in D_{J}\right\}$ by 4.1. Furthermore $h_{k}\left(x_{J}\right) \neq 0$ and

$$
\begin{gathered}
h_{1}\left(x_{J}\right) K W \cong h_{1}\left(x_{J}\right) K W_{J} \otimes_{K W_{J}} K W, \\
h_{q}\left(x_{J}\right) K[W] \cong h_{q}\left(x_{J}\right) K\left[W_{J}\right] \otimes_{K\left[W_{J}\right]} K[W]
\end{gathered}
$$

with basis $\left\{h_{1}\left(x_{J}\right) \otimes w \mid w \in D_{J}\right\},\left\{h_{q}\left(x_{J}\right) \otimes T_{w} \mid w \in D_{J}\right\}$ respectively. Let $\chi$ be the character afforded by $X_{J}=x_{J} \mathscr{G}(W)$. Then, by the above, $\chi_{k}$ is the character afforded by $X_{J}^{(k)}$, and $X_{J}^{(1)} \cong X_{J}^{(q)}$ by construction of the identifying isomorphism $\tau$ : $K[W] \rightarrow K W$. Similarly $Y_{J}^{(1)} \cong Y_{J}^{(q)}$.

We need some results from the representation theory of symmetric groups. For details the reader is referred to [14]. Remember that there is a bijection between $M_{s}$ and the set of all inequivalent irreducible characters of $W$, denoted by $\lambda \leftrightarrow \phi^{\lambda}$ $\left(\lambda \in M_{s}\right.$ ). If $S^{\lambda}$ denotes the Specht module over $R$ corresponding to $\lambda \in M_{s}$, then $K S^{\lambda}$ affords $\phi^{\lambda}$. It may be constructed in the following way:

Let $\lambda_{\Lambda}=\lambda_{1} \geq \lambda_{2} \geq \cdots \geq \lambda_{k}$. Define $J=J_{\lambda}$ to be the set of basic transpositions $(i, i+1)$, where $\left(\sum_{j=1}^{m} \lambda_{j}\right)+1 \leq i \leq\left(\sum_{j=1}^{m+1} \lambda_{j}\right)-1$ for some $\Lambda \in \mathscr{F}, 0 \leq m \leq k-$ 1. Then $W_{\lambda}=W_{J}$ is just the standard Young subgroup of $W$ corresponding to $\lambda \in M_{s}$. Let $J(\Lambda)=J \cap \mathscr{T}(\Lambda)(\Lambda \in \mathscr{F})$. Let $t$ be the $\lambda_{\Lambda}$-tableau in which the numbers $\left\{1,2, \ldots, m_{\Lambda}(s)\right\}$ increase along the rows. Then $W_{\lambda}(\Lambda)=W_{J(\Lambda)}$ is just the row stabilizer of $t$. Let $C_{J}(\Lambda)$ be the column stabilizer of $t$ and set $C_{J}=\Pi C_{J}(\Lambda)$. Note that $x=h_{1}\left(x_{J}\right)$ is just the sum over the elements of $W_{J}=W_{\lambda}$. Let $y$ be the alternating sum over the elements of $C_{J}$, and set $x y R W=S^{\lambda}$. Then $K S^{\lambda}$ affords $\phi^{\lambda}$. Moreover, $x K W$ and $y K W$ have just one composition factor in common, namely $K S^{\lambda}$. 
This procedure cannot be transposed directly to Hecke (respectively generic) algebras, because the $K(u)$-subspace (K-subspace) of $\mathscr{G}(W)(K[W])$ generated by $\left\{T_{w} \mid w \in C_{J}\right\}$ is not a subalgebra in general. But $C_{J}$ is conjugate in $W$ to $W_{\lambda^{\prime}}$, where $\lambda^{\prime}=\Pi \lambda_{\Lambda}^{\prime}$ and $\lambda_{\Lambda}^{\prime}$ is the dual partition of $\lambda_{\Lambda}\left(\lambda=\Pi \lambda_{\Lambda}\right)$. So let us fix $w \in W$ such that $w^{-1} W_{\lambda^{\prime}} w=C_{J}$. Set $J^{\prime}=J_{\lambda^{\prime}}$ and define $\tilde{z}_{\lambda}=x_{J} T_{w^{\prime}}^{-1} y_{J^{\prime}} T_{w} \in \mathscr{G}(W)$, where $x_{J^{\prime}} y_{J^{\prime}}$ $\in \mathscr{G}[W]$ are defined as in 4.2. Finally, set $z_{\lambda}=h_{q}\left(\tilde{z}_{\lambda}\right) \in K[W]=K W$. Note that $T_{w^{\prime}}^{-1} \in \mathscr{G}[W]$. Inspecting the coefficients of $T_{v}{ }^{-1}(v \in \mathscr{T})$ we see that $T_{w} \otimes 1$ is invertible in $R[W] \subset K[W]$. So $z_{\lambda} \in R[W]$.

\subsection{Lemma. Assume $q^{d_{\Lambda}}>2$ for all $\Lambda \in I$. Then $z_{\lambda} K[W] \cong K S^{\lambda}$.}

Proof. First note that $h_{1}\left(\tilde{z}_{\lambda}\right)=x w^{-1} h_{1}\left(y_{J^{\prime}}\right) w=x y \neq 0$, where $x, y \in R W$ are defined as above, hence $\tilde{z}_{\lambda} \neq 0$. Now $x K W$ and $y K W$ have just one composition factor in common, namely $K S^{\lambda}$. Obviously $y K W \cong w y w^{-1} K W=Y_{J^{\prime}}^{(1)} \cong Y_{J^{\prime}}^{(,)}$by 4.3 , so the same is true for $x K W=X_{J}^{(1)} \cong X_{J}^{q}=h_{q}\left(x_{J}\right) K[W]$ and $Y_{J^{\prime}}^{(q)}=h_{q}\left(y_{J^{\prime}}\right) K[W]$. Consequently either $z_{\lambda}=h_{q}\left(x_{J}\right) h_{q}\left(y_{J^{\prime}}\right)=0$ or $z_{\lambda} K[W] \cong K S^{\lambda}$. Now $\tilde{z}_{\lambda} \in x_{J} \mathscr{G}[W]$ $=x_{J} \mathscr{G}\left[W_{J}\right] \otimes \mathscr{G}[W]$, and a basis of $x_{J} \mathscr{G}\left[W_{J}\right] \otimes \mathscr{G}[W]$ is given by $\left\{x_{J} \otimes T_{W} \mid w \in\right.$ $\left.D_{J}\right\}$, where $D_{J}$ is defined as in 4.3. Furthermore, it can easily be seen that the coefficients of $\tilde{z}_{\lambda}=\sum_{w \in D_{J}} x_{J} \otimes T_{w} r_{w}\left(r_{w} \in K(u)\right)$ are Laurent polynomials in $u$ with integer coefficients. In particular, $r_{1}=f(u) / u^{k}$ for some $k \in \mathbf{N}$ and a polynomial $f(u) \in K[u]$ with integer coefficients. Using the specialization $u \rightarrow 1$ we see that $f(1)=1$. Hence $f(q)=1 \bmod (q-1)$ (note that $q>2$ by assumption), in particular $f(q) \neq 0$. This implies $z_{\lambda} \neq 0$ as desired.

We will see later that the assumption in 4.4 is no real restriction for our purpose. So let us assume in the following that $q^{\operatorname{deg} \Lambda}>2$ for all $\Lambda \in \mathscr{F}$ with $m_{\Lambda}(s) \neq 0$.

Let us return now to the $K G$-module $K S^{G}$ defined in $\$ 2$. Recall that $\operatorname{End}_{R G}\left(S^{G}\right)$ $=R[W]$ by 3.6. For $\lambda \in M_{s}$ define the $A$-module $S_{\lambda}$ to be $z_{\lambda} R[W](S \otimes 1)=$ $z_{\lambda} R[W](S \otimes 1) A \leq S^{G} \cdot e$, where $A \subseteq R G$ is defined as in $\S 3$. Then $S_{\lambda} R G=z_{\lambda} \cdot S^{G}$ is an $R G$-submodule of $S^{G}$.

\subsection{Lemma. $S_{\lambda} K G$ is an irreducible $K G$-module affording $\chi_{s, \lambda}$.}

Proof. $S_{\lambda} K G=z_{\lambda} K[W](S \otimes 1) K G=e_{\lambda} \cdot K S^{G}$, where $e_{\lambda} \in K[W]$ is the unique primitive idempotent contained in the irreducible $K[W]$-module $z_{\lambda} K[W] \cong K S^{\lambda}$ by 4.4. So $S_{\lambda} K G$ is irreducible.

By $[15,6.24,8.6], R_{T}^{G}(s)=R_{L_{0}}^{G}(\theta)$, where $L_{0}=C_{G}(s)$ and $\theta=\pi^{L_{0}}$ setting $\pi=\tilde{\hat{s}}$, the lifting of $\hat{s}$ to the standard Borel subgroup of $L_{0}$. Now $R_{L_{0}}^{G}(\chi)$ is (up to sign) irreducible for every irreducible constituent $\chi$ of $\theta$. So we may assume $G=L_{0}$, that is $s \in Z(G)$, the center of $G$. Furthermore, $\theta=\hat{s} \otimes 1_{B_{0}}^{G}$, where $1_{B_{0}}$ denotes the trivial character of $B_{0}, \otimes$ the usual product of characters, and $s$ on the right-hand side is considered as the linear character of $G=L_{0}$. So we may assume that $s=1 \in G$. But this is just the classical case, where it is well known that $e_{\lambda} K S^{G}$ affords $\chi_{s, \lambda}$ (see e.g. $[4,13])$.

We are now prepared to prove the main technical lemma. So define $D^{\lambda}=$ $\bar{R} S^{\lambda} / J\left(\bar{R} S^{\lambda}\right), D_{\lambda}=\bar{R} S_{\lambda} / J\left(\bar{R} S_{\lambda}\right)$ and $E_{\lambda}=\bar{R}\left(z_{\lambda} S^{G}\right) / J\left(\bar{R}\left(z_{\lambda} S^{G}\right)\right)$. We have to be careful here. In fact, if $\bar{z}_{\lambda}$ denotes the image of $z_{\lambda} \in R[W]$ in $\bar{R}[W]$, the $R G$-modules 
$\bar{R}\left(z_{\lambda} S^{G}\right)$ and $\bar{z}_{\lambda}\left(\bar{R} S^{G}\right)$ are not isomorphic in general, although there always exists an epimorphism from $\bar{R}\left(z_{\lambda} S^{G}\right)$ onto $\bar{z}_{\lambda}\left(\bar{R} S^{G}\right)$. In particular, $\bar{R}\left(z_{\lambda} S^{G}\right)$ may not necessarily be a submodule of $\bar{R} S^{G}$ in general.

4.6. Lemma. Let $\lambda \in M_{s}$ and assume that $D^{\lambda}$ is a simple $\bar{R}[W]-$ module. Then $D_{\lambda}$ is a simple $\bar{R} S$-homogeneous $\bar{R} A$-module occurring in $E_{\lambda}$ e as a composition factor. Finally $E_{\lambda}$ is simple.

Proof. By 3.7, $S_{\lambda}=z_{\lambda} R[W](S \otimes 1)$ is $S$-homogeneous. Hence $\bar{R} S_{\lambda}$ and its epimorphic image $D_{\lambda}$ are $\bar{R} S$-homogeneous, too. Again by $3.7 D_{\lambda}$ is irreducible. Note that $M e \leq M_{\tilde{R} A}$ for $\tilde{R} \in\{\bar{R}, R, K\}$ and an arbitrary $\tilde{R} G$-module $M$. Furthermore, $\bar{R}\left(z_{\lambda} S^{G} e\right)=\left(\bar{R}\left(z_{\lambda} S^{G}\right)\right) e$, so $E_{\lambda} e$ is the $\bar{R} S$-homogeneous component of $E_{\lambda}$. Let $X \leq \bar{R}\left(z_{\lambda} S^{G}\right)$. Then $X e \leq \bar{R}\left(z_{\lambda} S^{G} e\right)=\bar{R}\left(z_{\lambda}(S \otimes 1) A\right)=\bar{R} S_{\lambda}$. By the above, $J\left(\bar{R} S_{\lambda}\right)$ is the unique maximal $\bar{R} A$-submodule of $\bar{R} S_{\lambda}$, hence $X e \leq J\left(\bar{R} S_{\lambda}\right)$ or $X e=\bar{R} S_{\lambda}$. But $\bar{R} S_{\lambda} \subset X e \subset X$ implies $\bar{R}\left(S_{\lambda} R G\right)=\bar{R}\left(z_{\lambda} S^{G}\right) \leq X$, because $\bar{R}\left(S_{\lambda} R G\right)=\left(\bar{R} S_{\lambda}\right) R G$. Therefore every proper submodule of $\bar{R}\left(z_{\lambda} S^{G}\right)$ is contained in the proper $\bar{R} A$-submodule

$$
J\left(\bar{R}\left(z_{\lambda} S^{G}\right) e\right) \oplus \bar{R}\left(z_{\lambda} S^{G}\right)(1-e)=J\left(\bar{R}\left(z_{\lambda} S^{G} e\right)\right)+\bar{R}\left(z_{\lambda} S^{G}\right)(1-e),
$$

hence the sum of all proper submodules of $\bar{R}\left(z_{\lambda} S^{G}\right)$, too. Thus $E_{\lambda}$ is irreducible, and $D_{\lambda}$ occurs as a composition factor in $E_{\lambda} e$ (in fact $D_{\lambda} \leq E_{\lambda} \cdot e / J\left(E_{\lambda} \cdot e\right)$ ).

REMARK. Note that $\bar{R}\left(z_{\lambda} S^{G} e\right)$, hence $D_{\lambda}$ and $E_{\lambda} \cdot e$, are contained in $\bmod _{\bar{R} \mathscr{A}}(\bar{R} \mathscr{A} / \bar{R} S) \subset \bmod _{\bar{R} \mathscr{A}}(\bar{R} A / \bar{R} S)$.

5. In the previous section, we have worked out the way to use the Specht modules of $K[W]$ for defining irreducible $R G$-modules. However, this only works after having achieved sufficient knowledge on the representation theory of $R[W]$, which at the moment is in the state of development. So we have to restrict ourselves to special cases.

5.1. Hypothesis. Let $s \in G$ be semisimple satisfying 2.4 and assume $r$ divides $q^{\operatorname{deg} \Lambda}-1$ for all $\Lambda \in \mathscr{F}_{\text {with }} m_{\Lambda}(s) \neq 0$.

5.2. REMARK. Let $s \in G$ be semisimple, and assume that the order of $s$ is prime to $r$. Then $s$ satisfies 2.4 by 2.1 and 2.3. Let $B$ be an $r$-block of $G$ with semisimple part $s \in G$. Then, by $[9,5.6], s$ satisfies 5.1 if $B$ has inertia index one. However, if $r$ divides $q-1$, that is, if $r$ divides the order of $Z(G)$, then 5.1 is satisfied for all semisimple $s \in G$, and the following will hold for all $r$-blocks of $G$. Note, if $s \in G$ satisfies 5.1 and has order prime to $r$, then there exists only one $r$-block $B=B_{s}$ of $G$ with semisimple part $s$. Furthermore, a Sylow $r$-subgroup of $C_{G}(s)$ is a defect group $\delta\left(B_{s}\right)$ of $B_{s}$ and all geometric conjugacy classes $(t)^{G}$ with $t=s y, y \in \delta\left(B_{s}\right)$, are contained in $B_{s}$, by [9]. These are all of the irreducible characters contained in $B_{s}$ and the set of characters in $(s)^{G}$ restricted to the set of $r^{\prime}$-elements of $G$ form a basis of the space of Brauer characters of $G$ contained in $B_{s}$. In particular, $B_{s}$ just contains $\left|M_{s}\right|$ many inequivalent irreducible Brauer characters.

Recall the definition of $z_{\lambda} \in R[W]$, and let $\bar{z}_{\lambda}$ again be the image of $z_{\lambda}$ in $\bar{R}[W]$. Note that the assumption in 4.4 is satisfied if $s \in G$ satisfies 5.1, because $r \geq 2$, so $z_{\lambda} \neq 0$ by 4.4 . 
5.3. LemMA. Assume 5.1. Then $\bar{R}[W]$ and $\bar{R} W$ may be identified canonically such that $\bar{z}_{\lambda} \in \bar{R}[W]$ generates the usual Specht module $\bar{R} S^{\lambda}$ of $\bar{R}[W]=\bar{R} W\left(\lambda \in M_{s}\right)$.

Proof. Let $\bar{T}_{w}$ denote the image of $T_{w} \in R[W]$ in $\bar{R}[W]$. Now 5.1 implies $q^{\operatorname{deg} \Lambda} \equiv 1 \bmod r$ and $q^{\operatorname{deg} \Lambda}-1 \equiv 0 \bmod r$ for $\Lambda \in \mathscr{F}$ with $m_{\Lambda}(s) \neq 0$. The defining relations for $R[W]$ show immediately that $\bar{T}_{w} \rightarrow w \in \bar{R} W$ induces an isomorphism between $\bar{R}[W]$ and $\bar{R} W$. Identifying $\bar{R}[W]$ and $\bar{R} W$ by this isomorphism,

$$
\bar{z}_{\lambda}=\overline{h_{q}\left(x_{J}\right) h_{q}\left(T_{w}^{-1} y_{J^{\prime}} T_{w}\right)}=\overline{h_{1}\left(x_{J}\right) h_{1}\left(w^{-1} y_{J^{\prime}} w\right)},
$$

where $J, J^{\prime} \subset \mathscr{T}, T_{w}, x_{J}, y_{J^{\prime}} \in \mathscr{G}[W]$ are defined as in 4.4 and $-: R[W] \rightarrow \bar{R}[W]$, respectively -: $R W \rightarrow \bar{R} W$ denotes the canonical epimorphism. Now, for $\bar{R} W$, it is well known (see e.g. [14]) that

$$
\bar{R}\left(h_{1}\left(x_{J}\right) h_{1}\left(w^{-1} y_{J^{\prime}} w\right) R W\right) \cong \overline{h_{1}\left(x_{J}\right) h_{1}\left(w^{-1} y_{J^{\prime}} w\right)} \bar{R} W
$$

Thus $\bar{R}\left(z_{\lambda} R[W]\right)=\bar{z}_{\lambda} \bar{R} W$.

Summarizing we get by 4.4 and 5.3

5.4. TheOREM. Assume 5.1. Then there exist isomorphisms $\tau: K[W] \rightarrow K W$ and $\bar{R}[W] \rightarrow \bar{R} W$ such that the following diagram commutes, where $\leadsto$ denotes the decomposition map via $R[W]$ and $R W$ respectively:

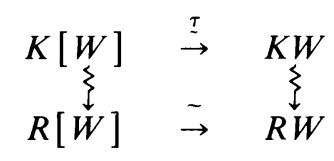

In particular, the decomposition matrices of $R[W]$ and $R W$ may be identified.

REMARK. We do not know if $R W \cong R[W]$ inducing the above isomorphisms. However, the theorem is trivially true in this case.

Theorem 5.4 says that we may apply the results from the representation theory of symmetric groups (extended to direct products of symmetric groups) to $R[W]$, as they are presented in the standard literature (see e.g. [14]). Remember that a partition $\lambda=\left(1^{r_{1}}, \ldots, k^{r_{h}}\right)$ of $k$ is called $r$-regular if $r_{i}<r$ for all $1 \leq i \leq k$. So call $\lambda=\Pi \lambda_{\Lambda} \in M_{s} r$-regular if $\lambda_{\Lambda}$ is $r$-regular for all $\Lambda \in \mathscr{F}$, otherwise $r$-singular. Let $M_{s, r^{\prime}}=\left\{\lambda \in M_{s} \mid \lambda r\right.$-regular $\}$ and $M_{s, r}=M_{s} \backslash M_{s, r^{\prime}}$. Denote by $\leq$ the dominance order on the set of partitions of $k \in \mathbf{N}$ as defined in [14,3.2], and extend this to $M_{s}$ setting $\lambda \leq \mu\left(\lambda=\Pi \lambda_{\Lambda}, \mu=\prod \mu_{\Lambda} \in M_{s}\right)$ if $\lambda_{\Lambda} \leq \mu_{\Lambda}$ for all $\Lambda \in \mathscr{F}$. Now, if $\lambda \in M_{s, r^{\prime}}$, the Specht module $\bar{R} S^{\lambda}$ (with respect to $\bar{R} W$ ) has a unique maximal submodule by [14, 4.9], hence the same is true if 5.1 holds for $\bar{R} S^{\lambda}$ as a $\bar{R}[W]$-module, by 5.4 , that is $D^{\lambda}$ is irreducible. Furthermore, $D^{\lambda} \neq D^{\mu}\left(\lambda, \mu \in M_{s, r^{\prime}}\right)$ if $\lambda \neq \mu$ and $\left\{D^{\lambda} \mid \lambda \in M_{s, r^{\prime}}\right\}$ is a full set of nonisomorphic irreducible $R[W]$-modules, again by 5.4. Finally, if $\left(a_{\lambda \rho}\right)\left(\lambda \in M_{s}, \rho \in M_{s, r^{\prime}}\right)$ denotes the decomposition matrix of $R W(R[W])$, then $a_{\rho \rho}=1$ and $a_{\lambda \rho} \neq 0$ implies $\rho \geq \lambda$.

Recall the definition of the $\bar{R} G$-module $E_{\lambda}\left(\lambda \in M_{s}\right)$. By the above and 4.6, $E_{\rho}$ ( $\left.\rho \in M_{s, r^{\prime}}\right)$ is irreducible if 5.1 holds. 
5.5. Lemma. Assume 5.1. Let $\rho, \rho^{\prime} \in M_{s, r^{\prime}}$ and $\rho \neq \rho^{\prime}$. Then $E_{\rho}$ and $E_{\rho^{\prime}}$ are two nonisomorphic irreducible $\bar{R} G$-modules.

Proof. By the above, $E_{\rho}$ and $E_{\rho^{\prime}}$ are irreducible, and, by 4.6 , the $\bar{R} A$-modules $D_{\rho}$, $D_{\rho^{\prime}}$ are irreducible, too. Furthermore, they are composition factors of $E_{\rho} e$ and $E_{\rho^{\prime}} e$ respectively. By $3.7, D_{\rho} \not D_{\rho^{\prime}}$. If $D_{\mu}\left(\mu \in M_{s, r^{\prime}}\right)$ occurs as a composition factor of $E_{\rho} e$, then it occurs as a composition factor of $\bar{R}\left(z_{\rho} S^{G} e\right)=R S_{\rho}$, because $E_{\rho}=$ $\bar{R}\left(z_{\rho} S^{G}\right) / J\left(\bar{R}\left(z_{\rho} S^{G}\right)\right)$. Thus, by $3.7, D^{\mu}$ occurs as a composition factor of $\bar{R} S^{\rho}$, forcing $\mu \geq \rho$. If $E_{\rho} \cong E_{\rho^{\prime}}$, then $E_{\rho} e \cong E_{\rho^{\prime}} e$, hence $D_{\rho}$ is a composition factor of $E_{\rho^{\prime}} e$ and $D_{\rho^{\prime}}$ of $E_{\rho} e$ by 4.6 . So, by the above, $\rho \leq \rho^{\prime}$ and $\rho^{\prime} \leq \rho$ forcing $\rho=\rho^{\prime}$, a contradiction.

Assume again 5.1 and let $b_{\lambda \rho}$ be the multiplicity of $E_{\rho}$ as a composition factor of $\bar{R}\left(z_{\lambda} S^{G}\right)$. Inspecting the $\bar{R} A$-module $\bar{R}\left(z_{\lambda} S^{G} e\right)$ and applying $3.7,4.6$ we conclude

5.6. Corollary. Let $\rho \in M_{s, r^{\prime}}, \lambda \in M_{s}$. Then $b_{\lambda \rho} \leq a_{\lambda \rho}$. In particular, $b_{\lambda \rho} \neq 0$ forces $\lambda \leq \rho$. Moreover, $b_{\rho \rho}=1$.

Assume 5.1. Let $i \in \bar{R}[W]=\bar{R} W$ be a primitive idempotent, and let $i \bar{R} W / J(i \bar{R} W) \cong D^{\rho}, \rho \in M_{s, r^{\prime}}$. Thus $Q^{\rho}=i \bar{R} W$ is a projective indecomposable $\bar{R} W$-module with head $D^{\rho}$. Let $Q_{\rho}=i\left(\bar{R} S^{G} e\right)=Q^{\rho}\left(\bar{R} S^{G} e\right)$. Then $Q_{\rho}$ is an indecomposable direct summand of $\bar{R} S^{G} e=(S \otimes 1) \bar{R} A$. Similarly $P_{\rho}=i\left(\bar{R} S^{G}\right)=Q^{\rho}\left(\bar{R} S^{G}\right)$ is an indecomposable direct summand of $\bar{R} S^{G}$ by 3.6. Note that $P_{\rho} e=Q_{\rho}$ and $P_{\rho} e \bar{R} G=Q_{\rho} \bar{R} G=P_{\rho}$.

5.7. Lemma. Assume 5.1, and let $\rho \in M_{s, r^{\prime}}$. Then $P_{\rho} / J\left(P_{\rho}\right)$ is simple and the head of $P_{\rho} / J\left(P_{\rho}\right) \cdot e$ is $D_{\rho}$.

Proof. Let $X \leq P_{\rho}$. Then $X e \leq P_{\rho} e=Q_{\rho}$, hence $X=P_{\rho}$ or $X e \leq J\left(Q_{\rho}\right)$. So all proper submodules of $P_{\rho}$ are contained in the $\bar{R} A$-submodule $J\left(Q_{\rho}\right)+P_{\rho} \cdot(1-e)$, hence $P_{\rho} / J\left(P_{\rho}\right)$ is irreducible. The canonical epimorphism $P_{\rho} \rightarrow P_{\rho} / J\left(P_{\rho}\right)$ induces an epimorphism $Q_{\rho}=P_{\rho} e \rightarrow P_{\rho} / J\left(P_{\rho}\right) \cdot e$, whose kernel is contained in $J\left(Q_{\rho}\right)$. Thus $D_{\rho}$, being the head of $Q_{\rho}$, is the head of $P_{\rho} / J\left(P_{\rho}\right) e$, too.

\subsection{Corollary. $P_{\rho} / J\left(P_{\rho}\right) \cdot e \cong D_{\rho}$.}

Proof. Let $1=i_{1}+\cdots+i_{k}$ be a decomposition of $1 \in \bar{R} W$ into a sum of primitive orthogonal idempotents and set $P_{j}=P_{i_{j}}(1 \leq j \leq k)$. Because $\bar{R}[W]=\bar{R} W$ $=\operatorname{End}_{\bar{R} G}\left(\bar{R} S^{G}\right), P_{j} \cong P_{m}$ if and only if $\rho_{j}=\rho_{m}, 1 \leq j, m \leq k$, where $i_{j} \bar{R} W / J\left(i_{j} \bar{R} W\right)$ $=D^{\rho_{1}}, 1 \leq j \leq m, \rho_{j} \in M_{s, r^{\prime}}$ suitable. 5.7 implies that an epimorphism $P_{j} \rightarrow P_{m}$ has to be bijective for $1 \leq i, j \leq m$, in particular $\rho_{j}=\rho_{m}$. Now it follows easily from Fitting's theorem (see e.g. $[11,5.2])$ that $J\left(i_{J} \bar{R} W\right) \cdot\left(\bar{R} S^{G}\right)$ is a proper $\bar{R} G$-submodule of $P_{j}$ for $1 \leq j \leq k$. By 5.7 this must be $J\left(P_{j}\right)$, and the corollary follows immediately.

6. Now we fix a semisimple element $s \in G$ of order prime to $r$ and assume that $r$ divides $q^{\operatorname{deg} \Lambda}-1$ for all $\Lambda \in \mathscr{F}$ with $m_{\Lambda}(s) \neq 0$. Then $s$ satisfies 5.1 and, by 5.2 , there exists only one $r$-block $B_{s}$ of $G$ with semisimple part $s$. Furthermore, $\delta\left(B_{s}\right)$ may be chosen as a Sylow $r$-subgroup of $C_{G}(s)$ and there are just $\left|M_{s}\right|$ nonisomorphic 
irreducible $\bar{R} G$-modules in $B_{s}$ by [9]. So far, we have determined $\left|M_{s, r^{\prime}}\right|$ many of them, namely $\left\{E_{\rho} \mid \rho \in M_{s, r^{\prime}}\right\}$, by 5.5.

Let $\delta\left(B_{s}\right)$ be a Sylow $r$-subgroup of $C_{G}(s), y \in \delta\left(B_{s}\right)$ and $t=s y$. We will vary $t$, thus all objects have to have the index $t$ in the following.

Note that $b_{t}$ may be considered as an $r$-block of $R L_{t}$ with semisimple part $s \in L_{t}$. Let the $R P_{t}$-module $S_{t}$ be defined as in 1.4 with $t$ replacing $s$ and $S_{t}$ replacing $S$. Our aim now is to find a sequence $1=y_{1}, y_{2}, \ldots, y_{k}$ of $r$-elements in $\delta\left(B_{s}\right)$ such that $t_{i}=s y_{i}$ satisfies 5.1 , and the resulting irreducible $\bar{R} G$-modules build a full set of nonisomorphic irreducible $\bar{R} G$-modules in $B_{s}$. But first we still need an auxiliary result. So let $t_{i}=s y_{i}(i=1,2)$ with $y_{i} \in \delta\left(B_{s}\right)$. Assume that $t_{i} \in G$ satisfies 2.4. Because $r$ divides $q^{\operatorname{deg} \Lambda}-1$ for all $\Lambda \in \mathscr{F}$ with $m_{\Lambda}(s) \neq 0$ by assumption, the same is true for $t_{i}$ instead of $s$, hence $t_{i}$ satisfies $5.1(i=1,2)$. Set $S_{i}=S_{t_{i}}, L_{i}=L_{t_{i}}$ and $P_{i}=P_{t_{i}}=L_{i} U_{i}$ with $U_{i}=U_{t_{i}}$, the Levi kernel of $P_{i}$. Let $\rho \in M_{t_{1}, r^{\prime}}, E=E_{\rho}$ and $\lambda \in M_{t_{2}}, M=\bar{R}\left(z_{\lambda} S_{2}^{G}\right)$. Note that $E$ is simple by 5.5. For an arbitrary group $H$ with subgroups $H_{1}$ and $H_{2}$ we write $H_{1} \leq{ }_{H} H_{2}$ if $H_{1}^{h} \leq H_{2}$ for some $h \in H$.

6.1. LEMMA. (i) If $E$ occurs as a composition factor of $\bar{R} S_{2}^{G}$ or $M$, then $L_{2} \leq_{G} L_{1}$.

(ii) If $E e_{t_{2}} \neq(0)$, then $L_{1} \leq_{G} L_{2}$.

Proof. First note that a Coxeter torus $T_{2}$ of $L_{2}$ is conjugate (in $G$ ) to a maximal torus of $L_{1}$ if and only if $L_{1} \leq{ }_{G} L_{1}$, because $L_{i}=\prod_{j=1}^{k} \mathrm{GL}_{\mu_{j}}(q)$ for a suitable partition $\mu=\mu_{1} \geq \mu_{2} \geq \cdots \geq \mu_{k}$ of $n$. Assume that $E$ occurs as a composition factor of $M$, then it occurs as a composition of $\bar{R} S_{2}^{G}$, because the composition factors of $\bar{R} S^{(1)}$ are independent of the choice of an $R G$-lattice $S^{(1)}$ in $K S^{G}$. By 4.6, $E \cdot e_{t_{1}} \neq(0)$, hence $\bar{R} S_{2}^{G} e_{t_{1}} \neq(0)$, and therefore $K S_{2}^{G} e_{t_{1}} \neq(0)$, that is, the trivial $K U_{1}$-module $I_{1}$ occurs as a composition factor of $K S_{2}^{G}$. If $E \cdot e_{t_{2}} \neq(0)$, then $\bar{R}\left(z_{\rho} \cdot S_{1}^{G}\right) e_{t_{2}} \neq(0)$, hence $z_{\rho} S_{1}^{G} e_{t_{2}} \neq(0)$. Thus the trivial $K U_{2}$-module $I_{2}$ occurs as a composition factor of $z_{\rho} K S_{1}^{G} \leq K S_{1}^{G}$ in this case, and it is enough to show (i). Taking characters and using Frobenius reciprocity we conclude in the first case:

$$
\begin{aligned}
0 & \neq\left(1_{U_{1}}, R_{T_{2}}^{G}\left(\hat{t}_{2}\right)\right)_{U_{1}}=\left(\tilde{\rho}^{G}, R_{T_{2}}^{G}\left(\hat{t}_{2}\right)\right)_{G} \\
& \left.=\sum_{T} \sum_{\theta \in \hat{T}} \varepsilon\left(\widetilde{R_{T}^{L_{1}}(\theta)}\right)^{G}, R_{T_{2}}^{G}\left(\hat{t}_{2}\right)\right)_{G} \\
& =\sum_{T} \sum_{\theta \in \hat{T}} \varepsilon\left(R_{T}^{G}(\theta), R_{T_{2}}^{G}\left(\hat{t}_{2}\right)\right)_{G},
\end{aligned}
$$

where $\rho$ denotes the regular representation of $L_{1}, T$ runs through all maximal tori of $L_{1}, \hat{T}$ denotes the set of irreducible characters of $T$ and $\varepsilon$ is a sign depending on $T$ and $L_{1}[15,6.23]$. By $[15,6.14] T_{2}$ has to be conjugate in $G$ to a maximal torus $T$ of $L_{1}$, forcing $L_{2} \leq_{G} L_{1}$.

Next let $y \in \delta\left(B_{s}\right)$ be arbitrary, $t=s y$. Then $C_{G}(t) \leq C_{G}(s)=\prod \mathrm{GL}_{m_{\Lambda}(s)}\left(q^{\operatorname{deg} \Lambda}\right)$. Let $\Pi C_{G}(t)_{\Lambda}, \Pi y_{\Lambda}$ and $\Pi t_{\Lambda}$ be the corresponding decompositions of $C_{G}(t), y$ and $t$ respectively. Let $\Lambda \in \mathscr{F}$ with $m_{\Lambda}(s) \neq 0$ and let $\tilde{\Gamma}$ be an elementary divisor of $y_{\Lambda}$. So $\tilde{\Gamma}$ is a monic irreducible polynomial with coefficients in $\operatorname{GF}\left(q^{\operatorname{deg} \Lambda}\right)$. Note that the order of $(\tilde{\Gamma}) \in \operatorname{GL}_{\operatorname{deg} \tilde{\Gamma}}\left(q^{\operatorname{deg} \Lambda}\right)$ is a power of $r$, so the order of each root $\omega$ of $\tilde{\Gamma}$ is a 
power of $r$, say $r^{k}$. Of course $\operatorname{GF}\left(q^{\operatorname{deg} \Lambda}\right)[\omega]=\operatorname{GF}\left(q^{\operatorname{deg} \Lambda \cdot \operatorname{deg} \Gamma}\right)=\operatorname{GF}(q)[\sigma \cdot \omega]$, where $\sigma$ is a root of $\Lambda$, because the order of $\sigma$ is prime to $r$, hence prime to the order of $\omega$. Let $\nu_{r}: \mathbf{Q} \rightarrow \mathbf{Z}$ denote the $r$-adic valuation and $\nu_{r}\left(q^{\operatorname{deg} \Lambda}-1\right)=j$. Set $i=k-j$ if $k \geq j$ and $i=0$ otherwise. Note that $k \geq 0$. It can easily be seen that $\operatorname{deg} \tilde{\Gamma}=r^{i}$ (compare e.g. [9, 3A]), hence the minimum polynomial $\Gamma$ of $\sigma \omega$ over $F$ has degree $\operatorname{deg} \Lambda \cdot r^{i}$, and over $\operatorname{GF}\left(q^{\operatorname{deg} \Lambda}\right)$ degree $r^{i}$. Replacing $G$ by $\mathrm{GL}_{m_{\Lambda}(s)}\left(q^{\operatorname{deg} \Lambda}\right) \leq$ $\mathrm{GL}_{m_{\Lambda}(s) \cdot \operatorname{deg} \Lambda}(q)$, we conclude that $m_{\Gamma}\left(t_{\Lambda}\right) \neq 0$ implies $\operatorname{deg} \Gamma=\operatorname{deg} \Lambda \cdot r^{i}$ for some $0 \leq i \in \mathbf{Z}$. Let $N_{i}=\left\{\Gamma \in \mathscr{F} \mid \operatorname{deg} \Gamma=\operatorname{deg} \Lambda \cdot r^{i}\right\}$ and $d_{i}=\sum_{\Gamma \in N_{i}} m_{\Gamma}\left(t_{\Lambda}\right)$. Then $m_{\Lambda}(s)=\sum_{i=0}^{\infty} d_{i} \cdot r^{i}$. Let $d_{\Lambda}$ be the sequence $\left(d_{i}\right)_{0 \leq i \in \mathbf{Z}}$ and $D_{\Lambda}$ be the set of sequences $\left(d_{i}\right)_{0 \leq i \in \mathbf{Z}}$ of natural numbers $d_{i}$ with $\sum_{i=0}^{\infty} d_{i} r^{i}=m_{\Lambda}(s)$. For the moment let $\lambda, \mu$ be partitions of some natural number $k, \lambda=\left(\lambda_{1} \geq \lambda_{2} \geq \cdots \geq \lambda_{j}\right)$, $\mu=\left(\mu_{1} \geq \mu_{2} \geq \cdots \geq \mu_{m}\right)$. Define the Levi subgroups $L_{\lambda}, L_{\mu}$ of $\mathrm{GL}_{k}(q)$ setting $L_{\lambda}=\prod_{i=1}^{j} \mathrm{GL}_{\lambda_{i}}(q), L_{\mu}=\prod_{i=1}^{m} \mathrm{GL}_{\mu_{i}}(q)$. On the set of partitions of $k$ define a partial order $\prec$ setting $\lambda \prec \mu$ if there exists a set partition $\left\{\Omega_{1}, \ldots, \Omega_{m}\right\}$ of $\{1, \ldots, j\}$ with $m$ parts (i.e. $\Omega_{i} \subseteq\{1, \ldots, j\}$, the $\Omega_{i}(1 \leq i \leq m)$ are pairwise disjoint and $\{1, \ldots, j\}=\bigcup_{i=1}^{m} \Omega_{i}$ ), such that $\mu_{i}=\sum_{h \in \Omega_{i}} \lambda_{h}$. Note that this is not the dominance order in general.

It is easy to see that $L_{\lambda} \leq_{\mathrm{GL}_{k}(q)} L_{\mu}$ if $\lambda \prec \mu$. So assume that $L_{\lambda}^{g} \leq L_{\mu}$ for some $g \in \mathrm{GL}_{k}(q)$. Let $T_{\lambda}$ be a Coxeter torus of $L_{\lambda}$. Then $T_{\lambda}^{g}$ is isomorphic to $\prod_{i=1}^{j} \mathrm{GF}\left(q^{\lambda_{i}}\right)$ and is a maximal torus of $L_{\mu}$, corresponding to a product $\Pi_{h=1}^{m} \nu^{(h)}$ of partitions $\nu^{(h)}$ of $\mu_{h}(1 \leq h \leq m)$. So, for each $h \in\{1, \ldots, m\}$, there exists a subset $\Omega_{h}$ of $\{1, \ldots, j\}$ such that $\sum_{i \in \Omega_{h}} \lambda_{i}=\nu^{(h)}$. Of course $\left\{\Omega_{1}, \ldots, \Omega_{m}\right\}$ is a set partition of $\{1, \ldots, j\}$. Thus we have shown that $L_{\lambda} \leq \mathrm{GL}_{k}(q) L_{\mu}$ if and only if $\lambda \prec \mu$.

Now let $D_{\Lambda}$ be as above. With $d_{\Lambda} \in D_{\Lambda}$ we may associate the proper partition $\left(\operatorname{deg} \Lambda^{d_{0}},(\operatorname{deg} \Lambda \cdot r)^{d_{1}}, \ldots,\left(\operatorname{deg} \Lambda \cdot r^{k}\right)^{d_{k}}\right)$ of $k=m_{\Lambda}(s) \cdot \operatorname{deg} \Lambda$. Denote this by $\lambda_{d_{\Lambda}}$ and notice that $d_{\Lambda} \rightarrow \lambda_{d_{\Lambda}}$ is an injective map. Define a partial order $\prec$ on $D_{\lambda}$ setting $d_{\Lambda} \prec d_{\Lambda}^{\prime}\left(d_{\Lambda}, d_{\Lambda}^{\prime} \in D_{\Lambda}\right)$ if $\lambda_{d_{\Lambda}} \prec \lambda_{d_{\Lambda}^{\prime}}$.

Let $D_{s}=\prod D_{\Lambda}$ (cartesian product) and define a partial order $\prec$ on $D_{s}$ setting $\Pi d_{\Lambda} \prec \Pi d_{\Lambda}^{\prime}\left(d_{\Lambda}, d_{\Lambda}^{\prime} \in D_{\lambda}\right.$ for $\left.\Lambda \in \mathscr{F}\right)$ if $d_{\Lambda} \prec d_{\Lambda}^{\prime}$ for all $\Lambda \in \mathscr{F}$. Finally, fix a linear order $\leq$ on $D_{s}$ compatible with $\prec$. As we have seen above, we have a map from $s \cdot \delta\left(B_{s}\right)$ into $D_{s}$. For $t=s y \in s \cdot \delta\left(B_{s}\right)$ let $\mathbf{d}_{t}$ be the image of $t$ under this map. Of course $D_{s}=\mathbf{d}_{1} \lesseqgtr \mathbf{d}_{2} \supsetneqq \cdots \lesseqgtr \mathbf{d}_{k}$ is finite, where $k=\left|D_{s}\right|$. If $\mathbf{d}_{t}=\mathbf{d}_{j}, t=s y \in s$. $\delta\left(B_{s}\right)$, call $j=\mathrm{ht}_{s}(y)$ the $s$-height of $y$. Later we will construct for each $j \in\{1, \ldots, k\}$ a $y_{j} \in \delta\left(B_{s}\right)$ of height $j$, such that in fact the map defined above is surjective.

6.2. Lemma. Assume that $s=m_{\Lambda}(s)(\Lambda)$, i.e. $s$ has only one elementary divisor, and let $y, y^{\prime} \in \delta\left(B_{s}\right), t=s y, t^{\prime}=s y^{\prime}$. Then $L_{t} \leq{ }_{G} L_{t^{\prime}}$ if and only if $\mathbf{d}_{t} \prec \mathbf{d}_{t^{\prime}}$. In particular $L_{t}=L_{t^{\prime}}$ if and only if $\mathrm{ht}_{s}(y)=\mathrm{ht}_{s}\left(y^{\prime}\right)$.

Proof. Obvious by the construction of $\mathbf{d}_{t}$ and $\mathbf{d}_{t^{\prime}}$ and the definition of $L_{t}$ and $L_{t^{\prime}}$.

Obviously the construction above may be extended to all $r$-elements $y$ of $C_{G}(s)$. If $y, y^{\prime} \in C_{G}(s)$ are $C_{G}(s)$-conjugate elements of $C_{G}(s), t=s y, t^{\prime}=s y^{\prime}$, then $\mathbf{d}_{t}=\mathbf{d}_{t^{\prime}}$ and ht ${ }_{s}(y)=\mathrm{ht}_{s}\left(y^{\prime}\right)$. Note that $t$ and $t^{\prime}$ are $G$-conjugate if and only if $y$ and $y^{\prime}$ are $C_{G}(s)$-conjugate. 
Fix again $\Lambda \in \mathscr{F}$ and let $1 \leq i \in \mathbf{Z}$. Let $\sigma$ be a root of $\Lambda$. Assume that the order of $\sigma$ is prime to $r$, and that $r$ divides $q^{\operatorname{deg} \Lambda}-1$. Let $m=\nu_{r}\left(q^{\operatorname{deg} \Lambda}-1\right), j=\operatorname{deg} \Lambda \cdot r^{i}$. Note that $\nu_{r}\left(q^{j}-1\right)=m+i$, because $r$ is odd. So fix an element $\omega=\omega_{i}$ of $\operatorname{GF}\left(q^{j}\right)$ of order $r^{m+i}$. Then $\operatorname{GF}\left(q^{\operatorname{deg} \Lambda}\right)[\omega]=\operatorname{GF}(q)[\sigma \cdot \omega]=\operatorname{GF}\left(q^{j}\right)$. Consequently the minimal polynomial $\Lambda_{i}$ of $\omega$ over $\operatorname{GF}\left(q^{\operatorname{deg} \Lambda}\right)$ has degree $r^{i}$, and the minimal polynomial $\Lambda_{i}^{\prime}$ of $\sigma \omega$ over $\operatorname{GF}(q)$ has degree $j$. Set $\Lambda_{0}=1, \Lambda_{0}^{\prime}=\Lambda$. Let $d_{\Lambda}=$ $\left(d_{i}\right)_{0 \leq i \in \mathbf{Z}} \in D_{\Lambda}$, where $s \in G$ is chosen as above. Then define $y_{\Lambda} \in C_{G}(s)_{\Lambda}$ setting $y_{\text {. }}=\prod_{i=0}^{\infty} d_{i}\left(\Lambda_{i}\right)$ (matrix direct sum).

For $\mathbf{d}=\prod d_{\Lambda} \in D_{s}$ set $y_{\mathbf{d}}=\prod y_{\Lambda} \in C_{G}(s)$. Note that $y$ is an $r$-element. Now, for $j \in\{1, \ldots, k\}, \mathbf{d}=\mathbf{d}_{j}$, choose $y_{j}=\delta\left(B_{s}\right) C_{G}(s)$-conjugate to $y_{\mathbf{d}}$, which is possible since we have chosen $\delta\left(B_{s}\right)$ to be a Sylow $r$-subgroup of $C_{G}(s)$. Set $t_{j}=s y_{j}$. In particular, $t_{1}=s$. Note that all elementary divisors of $t_{j}$ are of the form $\Lambda_{i}^{\prime}$ for some $0 \leq i \in \mathbf{Z}$, where $\Lambda$ runs through the set of elementary divisors of $s \in G$. The next lemma follows immediately from the definitions.

6.3. Lemma. Let $j, j^{\prime} \in\{1,2, \ldots, k\}$. Then $\mathbf{d}_{t_{1}}=\mathbf{d}_{j}$ and $\mathrm{ht}_{s}\left(y_{j}\right)=j$. In particular, the map $t \rightarrow \mathbf{d}_{t}$ from $s \cdot \delta\left(B_{s}\right)$ into $D_{s}$ is surjective. If $y_{j}^{g}=y_{j^{\prime}}$ for some $g \in C_{G}(s)$, then $j=j^{\prime}$. So this map induces a bijection between $\left\{t_{j} \mid 1 \leq j \leq k\right\}$ and $D_{s}$. Finally, if $\mathbf{d}_{j}=\prod d_{\Lambda}, d_{\Lambda}=\left(d_{i}\right)_{0 \leq i \in \mathbf{Z}}$, then $m_{\Lambda_{i}^{\prime}}\left(t_{j}\right)=d_{i}(0 \leq i \in \mathbf{Z})$.

In the following we will replace all occurring indices of the form $t_{j}(1 \leq j \leq k)$ by $j$ itself, e.g. $W_{j}=W_{t}, S_{j}=S_{t}$, etc. Note in particular that $M_{1, r^{\prime}}=M_{s, r^{\prime}}, M_{1}=M_{s}$ and $W_{1}=W_{s}$. Let $\lambda=\Pi \lambda_{\Lambda} \in M_{1}$, i.e. $\lambda_{\Lambda}$ is a partition of $m_{\Lambda}(s)(\Lambda \in \mathscr{F})$. Let $\lambda_{1}=\left(1^{k_{1}}, \ldots, m^{k_{m}}\right)$, where $m=m_{\Lambda}(s)$, i.e. $\sum_{\nu=1}^{m} k_{\nu} \cdot \nu=m$. Let $k_{\nu}=\sum_{i=0}^{\infty} d_{i}^{(\nu)} r^{i}$ be the $r$-adic expansion of $k_{\nu}$ for $1 \leq \nu \leq m$ and set $d_{i}=\sum_{\nu=1}^{m} d_{i}^{(\nu)} \cdot \nu(0 \leq i \in \mathbf{Z})$. Then $\sum_{i=0}^{x} d_{i} r^{i}=m=m_{\Lambda}(s)$, hence $d_{\Lambda}=\left(d_{i}\right)_{0 \leq i \in \mathbf{Z}} \in D_{\Lambda}$ and $\mathbf{d}=\Pi d_{\Lambda} \in D_{s}$, i.e. $\mathbf{d}=\mathbf{d}{ }_{j}$ for some $1 \leq j \leq k$. By 6.3, $m_{\Lambda_{i}^{\prime}}\left(t_{j}\right)=d_{i}$, and obviously $d_{i}=\sum_{\nu=1}^{m} d_{i}^{(\nu)} \nu=\sum_{\nu=1}^{d_{i}} d_{i}^{(\nu)} \nu$, thus $\left(1^{d_{i}^{(1)}}, \ldots, \nu^{d_{i}^{(n)}}, \ldots\right)=\lambda_{\Lambda_{i}^{\prime}}$ is an $r$-regular partition of $m_{\Lambda_{i}^{\prime}}\left(t_{j}\right)$. Taking the (cartesian) product over all $\Lambda \in \mathscr{F}$ and all $0 \leq i \leq \mathbf{Z}$ we have defined an element $\mu_{\lambda}$ of $M_{j, r^{\prime}}$. This defines a map $\lambda \rightarrow \mu_{\lambda}$ from $M_{1}$ into $\bigcup_{j=1}^{k} M_{j, r^{\prime}}$. Note that this union is disjoint by 6.3 .

On the other hand, let $\mu \in M_{j, r^{\prime}}, 1 \leq j \leq k$ and let $\Gamma$ be an elementary divisor of $t_{j}$. Then $\Gamma=\Lambda_{i}^{\prime}$ for some $0 \leq i \in \mathbf{Z}$ and some elementary divisor $\Lambda$ of $s$. Let $\mathbf{d}_{j}=\Pi d_{\Lambda}, d_{\Lambda}=\left(d_{i}\right)_{0 \leq i \in \mathbf{Z}}$, and let $\mu_{\Gamma}=\left(1^{d_{1}^{(1)}}, \ldots, \nu^{d_{i}^{(\nu)}}, \ldots\right)$, where $\mu=\Pi \mu_{\Gamma}$. By 6.3, $m_{\Gamma}\left(t_{j}\right)=d_{i}$, thus $\sum_{\nu=1}^{\infty} d_{i}^{(\nu)} \nu=d_{i}$. Define $k_{\nu}=\sum_{i=0}^{\infty} d_{i}^{(\nu)} r^{i}$ and note that $\sum_{\nu=1}^{x} \sum_{i=0}^{\infty} d_{i}^{(\nu)} r^{i} \nu=m_{\Lambda}(s)$, hence $\sum_{\nu=1}^{\infty} k_{\nu} \cdot \nu=m_{\Lambda}(s)$, i.e. $\lambda_{\mu}(\Lambda)=\left(1^{k_{1}}, \ldots, \nu^{k_{\nu}}, \ldots\right)$ is a partition of $m_{\Lambda}(s)$. Taking $\lambda_{\mu}=\Pi \lambda_{\mu}(\Lambda)$ we have defined a map $\mu \rightarrow \lambda_{\mu}$ from $\bigcup_{j=1}^{k} M_{j, r^{\prime}}$ into $M_{1}$, which is obviously the inverse of the map $\lambda \rightarrow \mu_{\lambda}$ defined above. So we have shown

6.4. Lemma. $\lambda \rightarrow \mu_{\lambda}$ defines a bijection between $M_{1}$ and the disjoint union $\bigcup_{j=1}^{k} M_{j, r^{\prime}}$.

In the following we will identify $M_{1}$ with $\bigcup_{j=1}^{k} M_{j, r^{\prime}}$ by this bijection.

We still need the following results:

6.5. Lemma. Let $T \leq G$ be a maximal torus of $G$ and $\theta$ a linear character of $T$. Let $u \in G$ such that the minimum polynomial of $u$ is $(X-1)^{n}$. Then $\left(R_{T}^{G}(\theta)\right)(u)=1$. 
Proof. $u$ is a unipotent element of $G$, in fact $u$ is conjugate to the matrix

$$
\left(\begin{array}{ccc}
1.1 & \ddots & 0 \\
0 & \ddots & 1 \\
& &
\end{array}\right) \text { (the Jordan Normalform of } u \text { ), }
$$

and the canonical rational form of $u$ is the companion matrix of the polynomial $(X-1)^{n}$.

So $\left(R_{T}^{G}(\theta)\right)(u)=Q_{T}^{G}(u)$, where $Q_{T}^{G}$ denotes the Green function of $G$ with respect to $T$ and $q$. If $T$ is not a Coxeter torus, the lemma follows easily from [15, 6.24]. If $T$ is a Coxeter torus, it follows from [15, 8.2]. Compare as well [10, Appendix].

Let $U_{0}$ be again the subgroup of all unipotent upper triangular matrices of $G$, and consider the restriction $R_{T}^{G}(\theta)_{U_{0}}$ of $R_{T}^{G}(\theta)$ to $U_{0}$. Because $R_{T}^{G}(\theta)$ is a virtual character of $G$, this restriction is a virtual character of $U_{0}$, i.e. a linear combination of irreducible characters of $U_{0}$ with integer coefficients. Let $m$ be the greatest common divisor of these coefficients. Because $\left(R_{T}^{G}(\theta)\right)(u)$ is an algebraic integer for all $u \in U_{0}$ we conclude

\subsection{Corollary. $m=1$.}

6.7. Lemma. Let $j \in\{1, \ldots, k\}$ and $y \in \delta\left(B_{s}\right) \cap L_{j}, t=s y$. Assume that $t^{g}=t_{j}$ for some $g \in G$. Then $t^{h}=t_{j}$ for some $h \in L_{j}$.

Proof. The elementary divisors of $t_{j}$ are of the form $\Lambda_{i}^{\prime}$, where $0 \leq i \in \mathbf{Z}$ and $\Lambda$ is an elementary divisor of $s$. Because $t$ and $t_{j}$ are conjugate in $G$, they have the same elementary divisors. Obviously we may assume $s=1$ (hence $r \mid q-1$ ). So $n=$ $\sum_{i=0}^{\infty} d_{i} r^{i}$ with $\mathbf{d}_{\Lambda}=\left(d_{i}\right)_{0 \leq i \in \mathbf{Z}}, \Lambda=X-1 \in \mathscr{F}$, hence $L_{j}=\prod_{i=0}^{\infty}\left(\mathrm{GL}_{r^{i}}(q)\right)^{d_{i}}$, and $\operatorname{deg} \Lambda_{i}^{\prime}=r^{i}$. Now the lemma follows immediately, because $y \in L_{j}$.

6.8. REMARK. If we replace $y_{j}$ by an arbitrary $y^{\prime} \in \delta\left(B_{s}\right)$, the lemma remains no longer true. The point is that we have fixed the polynomials $\Lambda_{i}^{\prime}$. For example, if we take $n=2, r \mid q-1, s=1 \in G$, and $w$ and $w^{\prime}$ as two different elements of order $r$ in $\mathrm{GF}(q), y=\left(\begin{array}{cc}w & 0 \\ 0 & w^{\prime}\end{array}\right), y^{\prime}=\left(\begin{array}{cc}w^{\prime} & 0 \\ 0 & w\end{array}\right)$, then $y \in L_{y^{\prime}}=\mathrm{GF}(q)^{*} \times \mathrm{GF}(q)^{*}, y \in \delta\left(B_{1}\right), y$ and $y^{\prime}$ are conjugate in $G$, but cannot be conjugate in the abelian group $L_{y^{\prime}}$.

Now we are prepared to prove the main technical lemma of this section.

\subsection{Lemma. Let $j \in\{1, \ldots, k\}$. Then $t_{j} \in G$ satisfies 5.1 .}

Proof. As we have seen, all elementary divisors $\Gamma$ of $t_{j}$ are of the form $\Lambda_{i}^{\prime}$, $0 \leq i \in \mathbf{Z}$, where $\Lambda$ is an elementary divisor of $s$. In particular, $\operatorname{deg} \Gamma=\operatorname{deg} \Lambda \cdot r^{i}$, hence $r$ divides $q^{\operatorname{deg} \Gamma}-1$ by $[9,3 \mathrm{~A}]$, because $r$ is odd dividing $q^{\operatorname{deg} \Lambda}-1$.

So we have to show that $t_{j}$ satisfies 2.4. For $j=1$, i.e. $t_{j}=s$, this is true by 2.1 and 2.3. So let $j>1$ and assume that $t_{j^{\prime}}$ satisfies 2.4 hence 5.1 for all $1 \leq j^{\prime}<j$. In order to prove 2.4(i) we have to consider $S_{j}$ as an $R L_{j}$-module contained in the block $b_{j}$ whose semisimple part is $s \in L_{j} \leq G$. We may restrict ourselves to a component $S_{j}(\Gamma)$ for some elementary divisor $\Gamma$ of $t_{j}$, so, using induction over $n$, we may assume 
that $j=k, n=\operatorname{deg} \Lambda \cdot r^{h}$ and $s=r^{h}(\Lambda)$ for some $h \in \mathbf{N}$. In particular, $t_{k}=\left(\Lambda_{h}^{\prime}\right)$, and $M_{k, r^{\prime}}=M_{k}$ contains one element $\lambda_{k}=\Pi \lambda_{\Gamma}$, with $\lambda_{\Gamma}=(1)$ for $\Gamma=\Lambda_{h}^{\prime}$ and $\lambda_{\Gamma}=(-)$ otherwise. By 5.5 and 6.4, for each $\mu \in M_{s}=M_{1}=\bigcup_{j=1}^{k} M_{j, r^{\prime}}$ with $\mu \neq \lambda_{k}$, we have defined an irreducible $\bar{R} G$-module $E_{\mu}$. Let $\lambda_{k} \neq \mu, \mu^{\prime} \in M_{s}$ and assume $E_{\mu} \cong E_{\mu^{\prime}}$. Let $\mu \in M_{j, r^{\prime}}, \mu^{\prime} \in M_{j^{\prime}, r^{\prime}}$. Then 6.1 implies immediately $L_{j}=L_{j^{\prime}}$, hence $j=j^{\prime}$ by 6.2 and $\mu=\mu^{\prime}$ by 5.5 . So we have found all but one irreducible $\bar{R} G$-module contained in $b_{k}=B_{s}$. Let $E$ be the remaining irreducible $\bar{R} G$-module contained in $B_{s}$. By $6.1, E_{\mu}$ does not occur as a composition factor of $\bar{R} S_{k}$, where $\lambda_{k} \neq \mu \in M_{1}$. So all composition factors of $\bar{R} S_{k}$ are isomorphic to $E$. Let $m$ be the multiplicity of $E$ as a composition factor of $\bar{R} S_{k}$. Then $\bar{R} S_{k}$ restricted to $U_{0}$ is isomorphic to a direct sum of $m$ copies of $E_{U_{0}}$, because $U_{0}$ is an $r^{\prime}$-group. Consequently $K S_{k} \cong m \cdot K E$ as $U_{0}$-modules, where $E$ is an $R U_{0}$-module such that $\bar{R} E \cong E_{U_{0}}$. Let $\chi$ be the character afforded by $K E$. Then $\pm R_{T_{k}}^{G}\left(\hat{t}_{k}\right)_{U_{0}}=m \chi$, forcing $m=1$ by 6.6. Hence $\bar{R} S_{k}=E=$ $E_{\lambda_{h}}$ is irreducible, proving 2.4(i).

Returning to the general case we now have proved that $t_{j}$ satisfies 2.4(i) if $t_{j^{\prime}}$ satisfies 5.1 for all $1 \leq j^{\prime}<j$. So let $V \in b_{j}$ be an irreducible constituent of $K S_{j P_{j}}^{G}$, where $S_{j}$ is considered as a $P_{j}$-module. Let $\psi$ be the character afforded by $V$. Then, because the semisimple part of $b_{j}$ is $s, \psi=\tilde{\chi}$ for some $\chi \in(s y)^{L_{j}}$ and some $r$-element $y \in C_{I, y}(s)$, i.e. $\chi=\chi_{s y, \lambda}$ for some $\lambda \in M_{s y}$, where we take $M_{s y}$ with respect to $L_{j}$. Now $\chi_{s y, \lambda}$ is a constituent of $\pm R_{T_{s i}}^{L_{s}}(\widehat{s y})$, and $R_{T_{s i}}^{L_{j}}(\widehat{s y})=\left(\widehat{R_{T_{s y} y}^{L_{s y}}(\widehat{s y})}\right)^{L_{j}}$ by $[15,6.24]$. In particular, $R_{T^{\prime}}^{L_{l}}(\widehat{s y})$ is up to sign a proper character of $L_{j}$. The character afforded by $K S_{j P_{1}}^{G}$ is up to sign $R_{T_{i}}^{G}\left(\hat{t}_{j}\right)_{P_{i}}$. So $V$ is an irreducible constituent of $K S_{j P_{i}}^{G}$ if and only if

$$
\left(\widetilde{R_{T_{s i}}^{L_{1}}(\widehat{s y})}, R_{T_{j}}^{G}\left(\hat{t}_{j}\right)_{P_{i}}\right)=\left(R_{T_{s y}}^{G}(\widehat{s y}), R_{T_{j}}^{G}\left(\hat{t}_{j}\right)\right) \neq 0
$$

using Frobenius reciprocity and $[15,6.24]$. So $s y$ has to be conjugate to $t_{j}$ in $G$ by [15, 6.14], hence in $L_{j}$ by 6.7 , forcing $R_{T_{s, r}}^{L_{j}}(\widehat{s y})=R_{T_{j}}^{L_{j}}\left(\hat{t}_{j}\right)$, i.e. $V=K S_{j}$, as desired. So the lemma is proved by induction over $j$.

Let $\rho \in M_{1}=\bigcup_{j=1}^{k} M_{j, r^{\prime}}$. Then, by 6.9 and 5.5, $E_{\rho}$ is an irreducible $\bar{R} G$-module in $B_{s}$. Suppose that $s=m_{\Lambda}(s)(\Lambda)$ for some $\Lambda \in \mathscr{F}$. Then we conclude as in the proof of 6.9 from $6.1,6.2$ and 5.5 that $E_{\rho} \cong E_{\rho^{\prime}}\left(\rho, \rho^{\prime} \in M_{1}\right)$ implies $\rho=\rho^{\prime}$. So we get immediately from $[9,8 \mathrm{~A}]$ the following

6.10. Lemma. Assume that $s$ has only one elementary divisor. Then $\left\{E_{\rho} \mid \rho \in M_{1}\right\}$ is a full set of nonisomorphic irreducible $\bar{R} G$-modules in $B_{s}$.

For $\rho \in M_{j, r^{\prime}}$ let $P_{\rho} \leq R S_{j}^{G}$ be defined as in 5.7. Recall the definitions of the subalgebra $A_{j}=A_{t}$ of $R G$, and the irreducible $\bar{R} A_{j}$-module $D_{\rho}$.

6.11. Lemma. Suppose that $s$ has only one elementary divisor $\Lambda \in \mathscr{F}$, and let $1 \leq j \leq k, \rho \in M_{j, r^{\prime}}$. Then $P_{\rho} / J\left(P_{\rho}\right) \cong E_{\rho}$. In particular, $E_{\rho} \cdot e_{j}=D_{\rho}$ as $\bar{R} A_{j}$-modules. 
Proof. Let $E=P_{\rho} / J\left(P_{\rho}\right)$. Then, by 6.10 and 5.7, $E=E_{\rho^{\prime}}$ for some $\rho^{\prime} \in M_{1}$, say $\rho^{\prime} \in M_{j^{\prime}, r^{\prime}}$. Now $E_{\rho^{\prime}}$ is a composition factor of $\bar{R} S_{j}^{G}$, so $L_{j} \leq{ }_{G} L_{j^{\prime}}$ by 6.1. On the other hand, $E_{\rho^{\prime}} \cdot e_{j}=D_{\rho} \neq(0)$ by 5.8, hence $L_{j^{\prime}} \leq{ }_{G} L_{j}$ again by 6.1. Therefore $j=j^{\prime}$ by 6.2. By 4.6 $D_{\rho^{\prime}}$ is a composition factor of $E_{\rho^{\prime}} \cdot e_{j}=\left(P_{\rho} / J\left(P_{\rho}\right)\right) \cdot e_{j}$, so $D_{\rho}=D_{\rho^{\prime}}$ by 5.8. Therefore $\rho=\rho^{\prime}$ as desired.

Let $s$ again be an arbitrary semisimple $r^{\prime}$-element of $G$ satisfying 5.1. Let $\rho \in M_{j, r^{\prime}}, \lambda \in M_{j^{\prime}}, 1 \leq j, j^{\prime} \leq k$, and denote the multiplicity of $E_{\rho}$ as a composition factor of $\bar{R}\left(z_{\lambda} S_{j^{\prime}}^{G}\right)$ by $d_{\lambda \rho}$, the $\left(\left|M_{j^{\prime}}\right| \times\left|M_{j, r^{\prime}}\right|\right)$-matrix $\left(d_{\lambda \rho}\right)$ by $D_{j^{\prime} j}$ and the decomposition matrix of $R W_{j}$ by $D^{j}$.

6.12. THEOREM. Let $s$ be a semisimple $r^{\prime}$-element of $G$ such that $r$ divides $q^{\operatorname{deg} \Lambda}-1$ for all $\Lambda \in \mathscr{F}$ with $m_{\Lambda}(s) \neq 0$. Then the following holds:

(i) $\left\{E_{\rho} \mid \rho \in M_{1}\right\}$ is a full set of nonisomorphic irreducible $\bar{R} G$-modules in $B_{s}$.

(ii) Let $1 \leq j, j^{\prime} \leq k, \rho \in M_{j, r^{\prime}}, \lambda \in M_{j^{\prime}}$. Then $d_{\lambda \rho} \neq 0$ implies that $\mathbf{d}_{j^{\prime}} \prec \mathbf{d}_{j}$. In particular, if $j \lessgtr j^{\prime}$, then $D_{j^{\prime} j}=0$.

(iii) $D_{j j}=D^{j}$ for $j \in\{1, \ldots, k\}$.

Proof. Assume first that $s=m_{\Lambda}(s)(\Lambda)$ for some $\Lambda \in \mathscr{F}$. Then (i) is 6.10 and (ii) follows immediately from 6.1 and 6.2(i). So let $j=j^{\prime}$, and let $\bar{R}\left(z_{\lambda} S_{j}^{G}\right)=C_{1} \supsetneqq C_{2} \supsetneqq$ $\cdots \supsetneqq C_{m} \supsetneqq(0)$ be composition series of $\bar{R}\left(z_{\lambda} S_{j}^{G}\right), C_{i} / C_{i+1}=E_{i}$ for $1 \leq i \leq m$ setting $C_{m+1}=(0)$. Then, by $6.10, E_{i}=E_{\rho_{i}}$ for some $\rho_{i} \in M_{1}$. Obviously $C_{i} \cdot e_{j} / C_{i+1} e_{j}=\left(C_{i} / C_{i+1}\right) \cdot e_{j}=E_{j} \cdot e_{j}$. Let $i \in\{1, \ldots, m\}$ and assume that $E_{i} e_{j} \neq$ (0). Then 6.1 implies immediately that $\rho_{i} \in M_{j, r^{\prime}}$, hence $C_{i} e_{j} / C_{i+1} e_{j}=E_{i} e_{j}=D_{\rho_{i}}$ is simple by 6.11 . This shows that $C_{1} e_{j} \geq C_{2} e_{j} \geq \cdots \geq C_{m} e_{j} \geq(0)$ is a composition series of $\bar{R}\left(z_{\lambda} S_{j}^{G}\right) e_{j}$. The standard basis theorem for Specht modules [14, 8.5] implies immediately that $\bar{R}\left(z_{\lambda} S_{j}^{G}\right) e_{j}=z_{\lambda}\left(\bar{R} S_{j}^{G} e_{j}\right) \leq \bar{R} S_{j}^{G} e_{j}$ is a reduction $\bmod r$ of the irreducible $K A_{j}$-module $z_{\lambda}\left(K S_{j}^{G} e_{j}\right)$. Counting multiplicities and applying 3.7, (iii) follows.

Now let $s=\prod m_{\Lambda}(s)(\Lambda) \in G$ again be arbitrary. Define the Levi subgroup $\tilde{L}$ of $G$ setting $\tilde{L}=\Pi \mathrm{GL}_{m_{\Lambda}(s) \cdot \operatorname{deg} \Lambda}(q)$, the parabolic subgroup $\tilde{P}$ of $G$ to be $\tilde{L} U_{0}$, and let $\tilde{U}$ be the unipotent radical of $\tilde{P}$. Then $\tilde{L}$ contains $C_{G}(s)$ and all $L_{j}(1 \leq j \leq k)$, thus $M_{i}$ with respect to $\tilde{L}$ and $M_{i}$ with resepect to $G$ coincide. Let $\tilde{b}$ be the block of $R \tilde{L}$ with semisimple part $s$ lifted to $\tilde{P}$. Note that we may extend all results developed so far to parabolic subgroups of general linear groups, as long as we restrict ourselves to blocks containing the unipotent radical of the parabolic subgroup in the kernel. In particular, we may apply it to $\tilde{b}$. Note as well that the theorem holds for $\tilde{b}$ by the construction of $\tilde{L}$ and $\tilde{P}$, and by the above. So, for $j, j^{\prime} \in\{1, \ldots, k\}, \rho \in M_{j . r^{\prime}}$, $\lambda \in M_{j^{\prime}}$, we denote by $\tilde{S}_{j}, \tilde{E}_{\rho}, \tilde{P}_{\rho}, \tilde{D}_{j^{\prime} j}$ the corresponding objects taken with respect to $\tilde{P}$.

Let $1 \leq i \leq k$. Then $\tilde{R} S_{i}^{G} \cong \sum_{g} \tilde{R} S_{i}^{\tilde{P}} \otimes g$ as $\tilde{R}$-space $(\tilde{R} \in\{\bar{R}, R, K\})$, where $g$ runs through a set of coset representatives of $\tilde{P}$ in $G$. Note that $\tilde{R}\left[W_{i}\right]\left(S_{i} \otimes 1\right) \leq \tilde{R} S_{i}^{\tilde{P}}$, and that $\tilde{R}\left[W_{i}\right]=$ End $_{\tilde{R} \tilde{P}}\left(\tilde{R} S_{i}^{\tilde{P}}\right)$. So, if $V$ is an arbitrary $\tilde{R} \tilde{P}$-submodule of $\tilde{R} S_{j}^{\tilde{P}}=$ $\left(\tilde{R} S_{j} \otimes 1\right) \tilde{R} P$, then $V \tilde{R} G \cong V^{G}$ canonically. Consider

$$
\tilde{P}_{\rho}=Q_{\rho} \bar{R} \tilde{P}=Q^{\rho}\left(\bar{R} S_{j}^{\tilde{P}} e_{j}\right) \bar{R} \tilde{P}=Q^{\rho} \bar{R} S_{j}^{\tilde{P}},
$$


where $Q_{\rho}$ and $Q^{\rho}$ are defined as in 5.7. Then $\tilde{P}_{\rho}^{G}=\left(Q^{\rho} \bar{R} S_{j}^{\tilde{P}}\right)^{G}=Q^{\rho}\left(\bar{R} S_{j}^{G}\right)=P_{\rho}$ and similarly $J\left(\tilde{P}_{\rho}\right)^{G}=J\left(P_{\rho}\right)$. Thus $\tilde{P}_{\rho}^{G} / J\left(\tilde{P}_{\rho}\right)^{G}=P_{\rho} / J\left(P_{\rho}\right)$. By $6.11, \tilde{P}_{\rho} / J\left(\tilde{P}_{\rho}\right)=\tilde{E}_{\rho}$, and obviously $\tilde{P}_{\rho}^{G} / J\left(\tilde{P}_{\rho}\right)^{G}=\tilde{E}_{\rho}^{G}$. Now $P_{\rho} / J\left(P_{\rho}\right)=\tilde{E}_{\rho}^{G}$ is simple by 5.7. Since all composition factors of $R S_{i}^{\tilde{P}}$ are of the form $\tilde{E}_{\mu}$ for some $\mu \in M_{1}, V \mapsto V^{G}$ defines an isomorphism from the submodule lattice of $\bar{R} S_{i}^{\tilde{P}}$ onto the submodule lattice of $\bar{R} S_{i}^{G}$. So all composition factors of $\bar{R} S_{i}^{G}$ are of the form $\tilde{E}_{\mu}^{G}$. From [9,8A] we conclude that $\left\{\tilde{E}_{\mu}^{G} \mid \mu \in M_{1}\right\}$ is a complete set of nonisomorphic irreducible $\bar{R} G$-modules in $B_{s}$. So, for an arbitrary $\bar{R} \tilde{P}$-module $V_{0}$ in $\tilde{b}, V \mapsto V^{G}$ defines an isomorphism from the submodule lattice of $V_{0}$ onto the submodule lattice of $V_{0}^{G} \in B_{s}$.

In particular, $E_{\rho} \cong \tilde{E}_{\mu}^{G}$ for some $\mu \in M_{1}$, say $\mu \in M_{i, r^{\prime}}, 1 \leq i \leq k$. Thus $\tilde{E}_{\mu}^{G}$ is a composition factor of $\bar{R} S_{j}^{G}$, hence $\tilde{E}_{\mu}$ of $\bar{R} S_{j}^{\tilde{p}}$ by the above, forcing $\mathbf{d}_{j} \prec \mathbf{d}_{i}$, since the theorem holds for $\tilde{b}$. On the other hand $L_{j}$ and $L_{i}$ must be conjugate in $G$ by 6.1 . From the definition of the partial order $\prec$ we get immediately $i=j$. As in the proof of 6.11 we conclude $\rho=\mu$, i.e. $\tilde{E}_{\rho}^{G}=E_{\rho}$. Now

$$
z_{\lambda} S_{j^{\prime}}^{G}=\left(z_{\lambda} S_{j^{\prime}}^{\tilde{P}}\right)^{G} \quad \text { and } \quad \bar{R}\left(z_{\lambda} S_{j^{\prime}}^{G}\right)=\bar{R}\left(z_{\lambda} S_{j^{\prime}}^{\tilde{P}}\right)^{G} .
$$

Thus the multiplicity of $\tilde{E}_{\rho}$ in $\bar{R}\left(z_{\lambda} S_{j^{\prime}}^{\tilde{P}}\right)$ equals the multiplicity of $E_{\rho}$ in $\bar{R}\left(z_{\lambda} S_{j^{\prime}}^{G}\right)$. This proves the theorem.

We have also shown

6.13. Corollary. Let $s$ and $\tilde{L}$ be as above, and b be the block of $\tilde{L}$ with semisimple part $s$. Then the decomposition matrices of $B_{s}$ and $b$ coincide.

Proof. Since $C_{G}(s)$ is contained in $\tilde{L}$ and $\delta\left(B_{s}\right)$ may be chosen in $C_{G}(s), \delta\left(B_{s}\right)$ is a defect group of $b$ as well. Moreover the geometric conjugacy classes in $B_{s}$ and $b$ are the same, because $t^{g}=t^{\prime}\left(t=s y, t^{\prime}=s y^{\prime}, y, y^{\prime} \in \delta\left(B_{s}\right)\right)$, for some $g \in G$ implies $g \in C_{G}(s) \leq \tilde{L}$. So the sizes of the decomposition matrices are the same. If $y \in \delta\left(B_{s}\right)$, $t=s y, \lambda \in M_{t}$ and $\rho \in M_{1}$, we conclude as in 6.12 that the multiplicity of $\tilde{E}_{\rho}$ in $\bar{R}\left(z_{\lambda} S_{t}^{\tilde{P}}\right)$ equals the multiplicity of $E_{\rho}$ in $\bar{R}\left(z_{\lambda} S_{t}^{G}\right)=\left(\bar{R}\left(z_{\lambda} S_{t}^{\tilde{P}}\right)\right)^{G}$, where $\tilde{P}$ and $\tilde{b}$ are defined as in 6.12. Thus the decomposition matrices of $B_{s}$ and $\tilde{b}$ coincide. However the same is true with $b$ instead of $\tilde{b}$.

Next let $y$ be an arbitrary $r$-element of $C_{G}(s), t=s y$. Arguing as in the proof of 6.9 with $y$ instead $y_{j}(1 \leq j \leq k)$, we see that $\bar{R} S_{t}$ is irreducible, in fact $\bar{R} S_{t}=\bar{R} S_{j}$ with $j=\mathrm{ht}_{s}(y)$.

\subsection{Lemma. $t$ satisfies $2.4(\mathrm{i})$ and $\bar{R} S_{t}=\bar{R} S_{j}$, where $j=\mathrm{ht}_{s}(y)$.}

REMARK. It is not true in general that $t$ satisfies 2.4(ii) too, as the example in 6.8 shows. In fact, here $\operatorname{End}_{K G}\left(K S_{y}^{G}\right)=K$, whereas End ${ }_{\bar{R} G}\left(\bar{R} S_{y}^{G}\right)=\bar{R}\left[W_{1}\right]$, and $W_{1}$ is isomorphic to the symmetric group on two letters.

As an easy consequence we get

6.15. TheOREM. Assume that $r$ divides $q-1=|Z(G)|$ and let $\chi$ be an irreducible cuspidal character of $G$. Then $\chi$, restricted to the $r^{\prime}$-elements of $G$, is an irreducible Brauer character. 
Summarizing we get the following theorem.

6.16. TheOREM. Let $s \in G$ be semisimple and assume that $r$ divides $q^{\operatorname{deg} \Lambda}-1$ for all elementary divisors of $s$. Then there exist $1=y_{1}, y_{2}, \ldots, y_{k} \in \delta\left(B_{s}\right)$ and a bijection between $M_{s}$ and the disjoint union $\bigcup_{j=1}^{k} M_{j, r^{\prime}}$ such that $\left\{E_{\rho} \mid \rho \in M_{j, r^{\prime}}, 1 \leq j \leq k\right\}$ is a full set of nonisomorphic irreducible $\bar{R} G$-modules in $B_{s}$. Ordering $\{1, \ldots, k\}$ downwards, the decomposition matrix $D$ of $B_{s}$ has the form

$$
D=\left(\begin{array}{ccc}
D^{k} & & \\
& D^{k-1} & 0 \\
* & & D^{1} \\
* & * & *
\end{array}\right),
$$

where $D^{j}(1 \leq j \leq k)$ denotes the decomposition matrix of $R W_{j}$.

Furthermore, the irreducible characters and Brauer characters in $B_{s}$ may be ordered such that $D$ has the (lower triangular) form

$$
D=\left(\begin{array}{lll}
1 & & \\
* & \ddots & 0 \\
& & 1 \\
* & * & *
\end{array}\right) .
$$

Proof. For both presentations of $D$ order the set of irreducible Brauer characters by ordering $\{1, \ldots, k\}$ downwards and $M_{j, r^{\prime}}$ lexicographically downwards. In the first case take the set $\left\{\chi_{t_{i}, \lambda} \mid 1 \leq j \leq k, \lambda \in M_{j}\right\}$ ordered analogously first, then the other irreducible characters in $B_{s}$. In the second case take $\left\{\chi_{t_{j}, \lambda} \mid 1 \leq j \leq k, \lambda \in M_{j, r^{\prime}}\right\}$ ordered in the same way first, then the remaining irreducible characters in $B_{s}$. Now the theorem follows from 6.16 and [14].

6.17. CoRollaRY. Let $G=\mathrm{GL}_{n}(q)$ for some natural number $n$ and some prime power $q$, and let $r$ be an odd prime not dividing $q-1$. Then the $r$-decomposition matrix of $G$ is lower unitriangular.

\section{REFERENCES}

1. C. T. Benson and C. W. Curtis, On the degrees and rationality of certain characters of finite Chevalley groups, Trans. Amer. Math. Soc. 165 (1972), 251-273; 202 (1975), 405-406.

2. N. Bourbaki, Groupes et algèbres de Lie, Chapitres 4-6, Hermann, Paris, 1968.

3. R. W. Carter, Simple groups of Lie type, Wiley, New York, 1972.

4. C. W. Curtis, N. Iwahori and R. Kilmoyer, Hecke algebras and characters of parabolic type of finite groups with $(B, N)$ pairs, Inst. Hautes Etudes Sci. Publ. Math. 40 (1972), 81-116.

5. C. W. Curtis and I. Reiner, Methods of representation theory with applications to finite groups and orders, Vol. 1, Wiley, New York, 1981.

6. E. C. Dade, Group-graded rings and modules, Math. Z. 174 (1980), 241-262.

7. P. Deligne and G. Lusztig, Representations of reductive groups over finite fields, Ann. of Math. (12) 103 (1976), 103-161.

8. W. Feit, Representations of finite groups, Part I, Yale Univ., 1969.

9. P. Fong and B. Srinivasan, The blocks of finite general and unitary groups, Invent. Math. 69 (1982), $109-153$. 
10. J. A. Green, The characters of the finite general linear groups, Trans. Amer. Math. Soc. 80 (1955), $402-447$. , Vorlesungen über Modulare Darstellungstheorie Endlicher Gruppen, Vorlesungen aus dem Mathematischen Institut Giessen, Heft 2, 1974.

12. R. B. Howlett and G. I. Lehrer, Induced cuspidal representations and generalized Hecke rings, Invent. Math. 58 (1980), 37-64.

13. N. Iwahori, On the structure of the Hecke ring of a Chevalley group over a finite field, J. Fac. Sci. Univ. Tokyo Sect. 1A Math. 10 (1964), 215-236.

14. G. D. James, The representation theory of the symmetric groups, Lecture Notes in Math., vol. 682 , Springer, Berlin and New York, 1978.

15. B. Srinivasan, Representations of finite Chevalley groups, Lecture Notes in Math., vol. 764, Springer, Berlin and New York, 1979.

\section{Mathematisches Institut der Universität ESSEN, 4300 EsSen, West Germany}

Current address: Department of Mathematics/Computer Science, Clark University, Worcester, Massachusetts 01610 